\title{
Loss of Tiparp Results in Aberrant Layering of the Cerebral Cortex
}

\author{
Giulia Grimaldi, ${ }^{1 *}$ Barbora Vagaska, ${ }^{1 *}$ Oleksandr levglevskyi, ${ }^{1}$ Elena Kondratskaya, ${ }^{1}$ Joel C. Glover, ${ }^{1}$ and \\ Jason Matthews ${ }^{1,2}$
}

https://doi.org/10.1523/ENEURO.0239-19.2019

${ }^{1}$ Institute of Basic Medical Sciences, University of Oslo, Oslo 0372, Norway and ${ }^{2}$ Department of Pharmacology and Toxicology, University of Toronto, Toronto, Ontario M5S 1A8, Canada

\begin{abstract}
2,3,7,8-tetrachlorodibenzo-p-dioxin (TCDD)-inducible poly-ADP-ribose polymerase (TIPARP) is an enzyme that adds a single ADP-ribose moiety to itself or other proteins. Tiparp is highly expressed in the brain; however, its function in this organ is unknown. Here, we used Tiparp ${ }^{-1-}$ mice to determine Tiparp's role in the development of the prefrontal cortex. Loss of Tiparp resulted in an aberrant organization of the mouse cortex, where the upper layers presented increased cell density in the knock-out mice compared with wild type. Tiparp loss predominantly affected the correct distribution and number of GABAergic neurons. Furthermore, neural progenitor cell proliferation was significantly reduced. Neural stem cells (NSCs) derived from Tiparp ${ }^{-1-}$ mice showed a slower rate of migration. Cytoskeletal components, such as $\alpha$-tubulin are key regulators of neuronal differentiation and cortical development. $\alpha$-tubulin mono-ADP ribosylation (MAR) levels were reduced in Tiparp ${ }^{-1}$ cells, suggesting that Tiparp plays a role in the MAR of $\alpha$-tubulin. Despite the mild phenotype presented by Tiparp ${ }^{-/-}$mice, our findings reveal an important function for Tiparp and MAR in the correct development of the cortex. Unravelling Tiparp's role in the cortex, could pave the way to a better understanding of a wide spectrum of neurological diseases which are known to have increased expression of TIPARP.
\end{abstract}

Key words: cortex; cortex layering; mono-ADP ribosylation; PARP; post-translational modification; Tiparp

\section{Significance Statement}

2,3,7,8-tetrachlorodibenzo-p-dioxin (TCDD)-inducible poly-ADP-ribose polymerase (TIPARP) is an enzyme which adds a single ADP-ribose moiety to itself or other proteins and is highly expressed in the brain. However, its function in this organ remains unknown. Here, we show that Tiparp affects neural progenitor cell proliferation and migration, and its loss leads to aberrant organization of the mouse cortex, predominantly by disrupting the correct distribution and number of GABAergic neurons. Cytoskeletal components, such as $\alpha$-tubulin, are key regulators of neuronal differentiation and cortical development, here, we show that Tiparp is part of a complex that interacts with $\alpha$-tubulin and plays a role in its mono-ADP ribosylation (MAR). Despite the mild phenotype presented by the Tiparp knock-out mouse our findings reveal an important function of MAR by this enzyme in the correct development of the cortex.

\section{Introduction}

2,3,7,8-tetrachlorodibenzo-p-dioxin (TCDD)-inducible poly-ADP-ribose polymerase (TIPARP; also known as, PARP7/ARTD14/RM1) is expressed in many different tis-

Received June 17, 2019; accepted October 10, 2019; First published November 8, 2019.

The authors declare no competing financial interests. sues, including liver, heart, spleen, brain, and reproductive organs (Ma et al., 2001). TIPARP is one of 17 members of the PARP family that have diverse cellular

Author contributions: G.G. and B.V. designed research; G.G., B.V., O.I., and E.K. performed research; G.G., B.V., and J.M. analyzed data; G.G. and J.M. wrote the paper; J.G. and J.M. contributed unpublished reagents/analytic tools. 
functions but share a conserved catalytic PARP domain. They use nicotinamide adenine dinucleotide $\left(\mathrm{NAD}^{+}\right)$as a substrate to transfer one or more molecules of ADPribose to specific amino acid residues on target proteins. TIPARP mono-ADP ribosylates itself and other proteins including, TANK-binding protein 1 (TBK1) and the aryl hydrocarbon receptor (AHR; MacPherson et al., 2013; Ahmed et al., 2015). TIPARP regulates the pluripotency of embryonic stem cells, mediates Type I interferondependent antiviral responses, regulates autophagy and acts as a transcriptional repressor (MacPherson et al., 2013; Roper et al., 2014; Yamada et al., 2016; Han et al., 2018). It contains an $\mathrm{N}$-terminus, followed by a $\mathrm{CCCH}-$ zinc finger domain, a WWE (tryptophan-tryptophan-glutamate) domain, and the conserved PARP catalytic domain. Within the $\mathrm{N}$-terminus there is a short nuclear localization sequence which, along with the zinc finger domain, ensures that TIPARP is predominantly found in the nucleus; however, localization of TIPARP varies among cell lines and during different stages of the cell cycle (Vyas et al., 2013; Gomez et al., 2018). In HeLa cells, TIPARP has a diffuse cytoplasmic localization during mitosis, but it forms nuclear foci during interphase (Vyas et al., 2013). Moreover, in response to viral infection, reactive oxygen species (ROS), and mitochondrial damage, TIPARP translocates from the nucleus to the cytosol (Kozaki et al., 2017).

AHR is a ligand responsive transcription factor predominantly studied for its ability to mediate toxic responses of environmental pollutants, such as TCDD (FernandezSalguero et al., 1996). However, it is activated by numerous endogenous ligands and plays important roles in immune function, inflammation, stem cell differentiation, neurologic development and behavior (Stockinger et al., 2014). It has previously been reported that TIPARP functions as part of a negative feedback loop regulating AHR activity and that Tiparp $^{-/}$mice exhibit an increased sensitivity to dioxin-induced toxicities primarily due to increased AHR activity (Hutin et al., 2018).

The effects of TCDD and AHR in neurons, brain and cognitive functions has been investigated in numerous studies (Powers et al., 2005; Hood et al., 2006; Nishijo et al., 2007; Collins et al., 2008; Lin et al., 2008; Kimura et al., 2017). TCDD exposure in rodents has been reported to perturb the dendritic morphology of neurons (Kimura et al., 2015), cortical layer formation (Mitsuhashi et al., 2010), and neurogenesis in cerebellar granule cells (Collins et al., 2008). Treated rodents also exhibit a distinct

This work was supported by the European Union Seventh Framework Program (FP7-PEOPLE-2013-COFUND) Grant n609020-Scientia Fellows (to G.G.) and by the Johan Throne Holst Foundation and the University of Oslo (J.M.).

${ }^{*}$ G.G. and B.V. contributed equally to this work.

Correspondence should be addressed to Jason Matthews at jason. matthews@medisin.uio.no or Giulia Grimaldi at giulia.grimaldi@medisin.uio.no.

https://doi.org/10.1523/ENEURO.0239-19.2019

Copyright (C) 2019 Grimaldi et al.

This is an open-access article distributed under the terms of the Creative Commons Attribution 4.0 International license, which permits unrestricted use, distribution and reproduction in any medium provided that the original work is properly attributed. decrease in behavioral flexibility, associate learning, memory, neuronal response to stimuli, locomotor activity and social behavior as well as increased anxiety. The majority of these effects are mediated by AHR, which has an important role in regulating developmental processes required for higher brain function in adulthood (Kimura and Tohyama, 2017). Recently, TCDD treatment was reported to promote migration of astrocytes in an Ahrdependent manner and affect GABAergic neurons resulting in their altered distribution in the medial prefrontal cortex, although the mechanism of action remains elusive (Nguyen et al., 2013; Chen et al., 2019). In Caenorhabditis elegans, ahr-1, the orthologue of $A H R$, is implicated in specifying GABAergic neuron cell fate (Huang et al., 2004).

In humans, children exposed to TCDD and TCDD-like environmental pollutants before birth have impaired higher cognitive functions, which is supported by another report linking exposure to AHR activating poly aromatic hydrocarbons with intelligence in children (Seegal, 1996; Perera et al., 2009).

Increased Tiparp expression was observed in rat brains during the developmental formation of the neural network and during long-term potentiation (LTP) in adults (Matsuo et al., 2000). This suggests that TIPARP functions in brain development and in neuronal plasticity in adulthood, and may represent a key enzyme involved in molecular cascades that lead to structural changes in synaptic connections. Moreover, TIPARP was identified as a highly upregulated protein following trace fear conditioning and in neurologic disorders, such as epilepsy (Steenland, 2010; Dachet et al., 2015). In an integrated multi-cohort transcriptional meta-analysis of neurodegenerative diseases, TIPARP was shown to be strongly upregulated independently of age ( $\mathrm{Li}$ et al., 2014). Despite several reports identifying the TIPARP gene or its product as being elevated in neuronal diseases, there are no studies that investigate the physiologic role of Tiparp in the brain. Unravelling its potential role in the brain could pave the way to better understanding a wide spectrum of neurologic diseases. Herein, we investigated the effects of loss of Tiparp in the mouse cortex.

\section{Materials and Methods}

\section{Animals}

All experiments were approved by the Norwegian Animal Research authority (FOTS ID 10420). Mice were housed in a 12/12 $\mathrm{h}$ light/dark cycle and had access to food and water ad libitum. The Tiparp ${ }^{-1-}$ mice where exon 3 of the Tiparp gene was removed were generated in C57BL/6N strain. A full description of the generation of these mice has been previously described (Hutin et al., 2018b). The animal colony was maintained by breeding heterozygotes and thus strain-specific wild-type $\left(\right.$ Tiparp $\left.^{+/+}\right)$ control mice or Tiparp ${ }^{-/}$mice were used in all experiments. Mice of either sex and wherever possible same sex littermates were used in these studies. 
Table 1. Primer sequences of the primers used for qPCR

\begin{tabular}{|c|c|c|}
\hline Gene name & Forward primer sequence & Reverse primer sequence \\
\hline Blbp & GGACACAATGCACATTCAAGAAC & CCGAACCACAGACTTACAGTTT \\
\hline$D c x$ & CCTTGATGGAAAGCAGGTCAC & GGCCTGGGCTITAGCAGAT \\
\hline Glast1 & ACCAAAAGCAACGGAGAAGAG & GGCATTCCGAAACAGGTAACTC \\
\hline Nestin & CCCTGAAGTCGAGGAGCTG & CTGCTGCACCTCTAAGCGA \\
\hline Nfh & GCCGCTTACAGAAAGCTCCT & ATGTGCGTGGATATGGAGGG \\
\hline Pax6 & TACCAGTGTCTACCAGCCAAT & TGCACGAGTATGAGGAGGTCT \\
\hline Pdgfr & AGAGTTACACGTTTGAGCTGTC & GTCCCTCCACGGTACTCCT \\
\hline Sox2 & ACAGAGAAAACCTGAGGGCG & GAAGCGCCTAACGTACCACT \\
\hline$T B P$ & GCACAGGAGCCAAGAGTGAA & TAGCTGGGAAGCCCAACTTC \\
\hline Tiparp & ACATCACACCGTATTGCCCT & GCCCAAAAGTCTTGTCCTCCAT \\
\hline Tubb3 & TGAGGCCTCCTCTCACAAGTA & CCGCACGACATCTAGGACTG \\
\hline
\end{tabular}

\section{RNA isolation and qPCR}

Mice were euthanized by open-drop exposure to isoflurane and cervical dislocation. The brains were extracted in ice-cold PBS, various brain regions were dissected and flash frozen in liquid nitrogen. The tissue was then homogenized in TRIzol reagent (Thermo Fisher Scientific) and total RNA and RNA from mouse neural stem cells (mNSCs) were isolated with Aurum Total RNA Mini kit (Bio-Rad) according to the manufacturer's instructions. All RNA was reverse transcribed using HighCapacity cDNA Reverse Transcription kit (Thermo Fisher Scientific). Transcript levels were quantified by qPCR using KAPA SYBR Fast Universal kit (Sigma-Aldrich). The amplified genes were normalized to tata-binding protein $(T b p)$. Primer sequences are provided in Table 1.

\section{Immunohistochemistry}

Mice were euthanized as described above and the brains were dissected in ice-cold PBS and then fixed overnight in $4 \%$ paraformaldehyde (PFA). Fixed brains were washed with PBS and incubated overnight in 30\% $(w / v)$ sucrose before embedding in Optimal Cutting Temperature compound (OCT). All mouse brain tissue was cryo-sectioned into 15- $\mu \mathrm{m}$-thick sections, which were air dried before use. Unless noted otherwise, sagittal section from comparable lateral regions within a third from the midline were used.

For Nissl staining, sections were placed in $0.1 \%$ cresyl violet solution for $10 \mathrm{~min}$ at $37^{\circ}$ before rinsing them in distilled water and then differentiated in 95\% ethyl alcohol for $15 \mathrm{~min}$. The sections were then dehydrated in 100\% alcohol twice for $5 \mathrm{~min}$. The sections were cleared in xylene twice for 5 min before mounting.

mNSCs were grown on poly-D-lysine coated glass cover slips, whereas COS- 1 cells were grown on noncoated cover slips. Both cell types were fixed for $15 \mathrm{~min}$ in $4 \%$ PFA before proceeding with immunofluorescence staining.

For immunofluorescence sections or cells were rinsed in PBS then blocked for $45 \mathrm{~min}$ in in $0.1 \%$ Triton X-100, $10 \%$ BSA in PBS at room temperature. Sections were then incubated overnight at $4^{\circ} \mathrm{C}$ with primary antibodies. They were washed in PBS and incubated at room tem- perature for $1 \mathrm{~h}$ with species-appropriate Alexa Fluor or cyanine dye conjugated secondary antibodies and Hoechst 33258 (Sigma) and mounted with Vectashield anti-fading mounting medium (Vectorlabs). The antibodies used are provided in Table 2. Images were obtained with a Zeiss LSM 710 confocal or Zeiss Axio Observer microscope. Immunohistochemistry images were analyzed with CellProfiler 3.0 software (www.cellprofiler.org; Broad Institute; Carpenter et al., 2006) and bin analysis, where the thickness of the cortex was divided into 40 equal intervals, was done with KNIME Analytics Platform (www.knime.com; Berthold et al., 2009)

\section{Electrophysiology}

For whole-cell patch-clamp experiments, mice (P14P21) were euthanized as described above, and the brain rapidly removed to ice-cold oxygenated $\left(95 \% \mathrm{O}_{2} / 5 \% \mathrm{CO}_{2}\right)$ artificial CSF (ACSF; $128 \mathrm{mM} \mathrm{NaCl}, 4 \mathrm{mM} \mathrm{KCl}, 21 \mathrm{mM}$ $\mathrm{NaHCO}_{3}, 0.5 \mathrm{mM} \mathrm{NaH} \mathrm{PO}_{4}, 30 \mathrm{mM}$ glucose, $1 \mathrm{mM}$ $\mathrm{MgCl}_{2}$, and $1 \mathrm{mM} \mathrm{CaCl}$; pH 7.2; 290-300 mOsm/l). Coronal brain slices of $350 \mu \mathrm{m}$ containing the cerebral cortex at the emergence of the dorsal hippocampus were cut using a vibrating microtome (VT1000S; Leica). Slices were stored and allowed to recover at room temperature in a submerged chamber in oxygenated standard ACSF (same composition as dissection ACSF, except that $\mathrm{CaCl}_{2}$ was at a concentration of $2 \mathrm{mM}$ ) for at least $1 \mathrm{~h}$ before experimentation. The slices were then transferred to a submersion chamber that was perfused with the oxygenated ACSF at $31^{\circ} \mathrm{C}$ for whole-cell recording. The cortex primary neurons were visually identified by their pyramidal shape and regular spikes with an up-right infrareddifferential interference contrast (IR-DIC) microscope (Olympus BX51WI) and captured with a CoolSNAP EZ with SSD ICX285 (Photometrics) video camera. Spontaneous IPSCs and EPSCs were recorded by using patch clamp technique in a whole-cell configuration. Patch electrodes were fabricated from borosilicate glass capillaries with an outer diameter of $1.5 \mathrm{~mm}$ (Sutter Instruments) using an automated puller (PC-10; Narishige). The internal pipette solution for studying excitatory synapses contained $130 \mathrm{mM} \mathrm{K}$-methylsulfate, $5 \mathrm{mM} \mathrm{NaCl}, 1 \mathrm{mM} \mathrm{CaCl}$, $20 \mathrm{mM}$ HEPES, $0.5 \mathrm{mM}$ EGTA, $3 \mathrm{mM} \mathrm{Mg}-\mathrm{ATP}$, and 0.4 
Table 2. List of primary and secondary antibodies used

\begin{tabular}{|c|c|c|c|c|c|c|c|}
\hline Antibody & RR ID & Target antigen & Manufacturer & Catalog number & Host & Clone & Dilution \\
\hline GFP & AB_300798 & GFP & Abcam & ab13970 & chicken & polyclonal & 1:3000 (IHC) \\
\hline GFP & AB_303395 & GFP & Abcam & $a b 290$ & rabbit & polyclonal & $1: 1000$ (WB) \\
\hline Parvalbumin & AB_2174013 & $\begin{array}{l}\mathrm{Ca}^{+2} \text {-binding site/ } \\
\text { specifically stains } \\
\text { the } \mathrm{Ca}^{+2} \text {-bound } \\
\text { form of parvalbumin }\end{array}$ & Millipore & MAB1572 & mouse & $\begin{array}{r}\text { monoclonal, IgG1, } \\
\text { clone PARV-19 }\end{array}$ & 1:500 (IHC) \\
\hline Calbindin & AB_10000340 & Calbindin D-28k & Swant & CВ 38 & rabbit & polyclonal & 1:1000 (IHC) \\
\hline Calretinin & AB_10000321 & Calretinin human & Swant & $7699 / 3 \mathrm{H}$ & rabbit & polyclonal & $1: 200(\mathrm{IHC})$ \\
\hline NeuN-biotin conjugated & AB_2298772 & $\begin{array}{l}\text { Neuron-specific } \\
\text { nuclear protein/ } \\
\text { FOX3/RBFOX3 }\end{array}$ & Millipore & MAB377B & mouse & monoclonal lgG1, clone $A 60$ & $1: 500(\mathrm{IHC})$ \\
\hline $\begin{array}{l}\text { Biotinylated } \\
\text { Wisteria } \\
\text { Floribunda } \\
\text { agglutinin (WFA) }\end{array}$ & $\mathrm{N} / \mathrm{A}$ & $\begin{array}{l}\text { N-acetylgalactosamine } \\
\text { (GalNAc) }\end{array}$ & Sigma & L1766 & $\mathrm{N} / \mathrm{A}$ & $\mathrm{N} / \mathrm{A}$ & $1: 100(\mathrm{IHC})$ \\
\hline GFAP & AB_10013382 & $\begin{array}{l}\text { Glial fibrillary } \\
\text { acidic protein }\end{array}$ & Dako & Z0334 & rabbit & polyclonal & $1: 400(\mathrm{IHC})$ \\
\hline TUBB3 & AB_262133 & Tubulin, $\beta$ III & Sigma & T2200 & rabbit & polyclonal & $1: 500(\mathrm{IHC})$ \\
\hline sox2 & $\mathrm{N} / \mathrm{A}$ & Transcription factor SOX-2 & Stem Cell Tech & 60055 & rabbit & polyclonal & $1: 100(\mathrm{IHC})$ \\
\hline $\begin{array}{l}\text { Nestin/radial } \\
\text { glial marker }\end{array}$ & AB_531887 & Nestin & DSHB & $\mathrm{RC} 2$ & mouse & $\begin{array}{l}\text { monoclonal, } \\
\operatorname{lgM}\end{array}$ & $1: 10(\mathrm{IHC})$ \\
\hline Vimentin & AB_531888 & Vimentin & DSHB & 3Св2 & mouse & monoclonal, IgM & $1: 10(\mathrm{IHC})$ \\
\hline Pax6 & AB_291612 & Pax6 & $\begin{array}{l}\text { Covance Research } \\
\text { Products }\end{array}$ & PRB-278P & rabbit & polyclonal & $1: 100(\mathrm{IHC})$ \\
\hline Pax6 & AB_528427 & Pax6 & DSHB & PAX6 & mouse & monoclonal lgG1 & $1: 10(\mathrm{IHC})$ \\
\hline CNP/Rip & AB_531796 & $\begin{array}{l}\text { 2', 3'-cyclic- } \\
\text { nucleotide } \\
\text { 3'-phosphodiesterase } \\
\text { (CNP) }\end{array}$ & DSHB & Rip & mouse & monoclonal, IgG1 & $1: 100(\mathrm{IHC})$ \\
\hline Synapsin $1 / 2$ & AB_1106784 & Synapsin $1 / 2$ & $\begin{array}{l}\text { Synaptic } \\
\text { Systems }\end{array}$ & 106004 & guinea pig & polyclonal & $1: 200(\mathrm{IHC})$ \\
\hline Ctip2 & AB_2064130 & BCL11B & Abcam & ab18465 & rat & polyclonal & $1: 500(\mathrm{IHC})$ \\
\hline Satb2 & AB_882455 & SATB2 & Abcam & ab51502 & mouse & monoclonal, lgG1 & $1: 200(\mathrm{IHC})$ \\
\hline$\alpha$-Tubulin & AB_477579 & $\alpha$-Tubulin & Sigma & T5168 & mouse & $\begin{array}{c}\text { monoclonal, IgG1, } \\
\text { clone B-5-1-2 }\end{array}$ & $\begin{array}{r}1: 500(\mathrm{IHC}), \\
1: 10000(\mathrm{WB}), \\
2.5 \mu \mathrm{g} / \mathrm{rxn}(\mathrm{IP})\end{array}$ \\
\hline$\beta$-Actin & AB_476697 & b-actin & Sigma & A2228 & mouse & $\begin{array}{l}\text { monoclonal, } \\
\text { IgG1, clone AC-74 }\end{array}$ & 1:10000 (WB) \\
\hline $\mathrm{pH} 3$ & AB_310177 & $\begin{array}{c}\text { Phospho-histone } \\
\text { H3 (Ser10) }\end{array}$ & Millipore & $06-570$ & rabbit & polyclonal & $1: 500(\mathrm{IHC})$ \\
\hline Acetylated $\alpha$-tubulin & AB_477585 & $\begin{array}{l}\alpha \text {-Tubulin, } \\
\text { acetylated } \\
\text { (Lys-40) }\end{array}$ & Sigma & T6793 & mouse & $\begin{array}{l}\text { monoclonal, } \\
\text { IgG2b, clone } \\
\text { 6-11B-1 }\end{array}$ & 1:1000 (WB) \\
\hline $\begin{array}{l}\text { Anti-mono-ADP- } \\
\text { ribose binding } \\
\text { reagent }\end{array}$ & AB_2665469 & $\begin{array}{l}\text { Mono } \\
\text { ADP-ribose }\end{array}$ & Millipore & MABE1076 & E.coli & monoclonal & 1:1000 (WB) \\
\hline Secondary antibody & RR ID & Conjugated & Manufacturer & $\begin{array}{l}\text { Catalog } \\
\text { number }\end{array}$ & Host & & \\
\hline Anti-mouse IgG & AB_330924 & HRP & $\begin{array}{l}\text { Cell } \\
\quad \text { Signaling }\end{array}$ & $7076 S$ & horse & & \\
\hline Anti-rabbit IgG & AB_2099233 & HRP & Cell Signaling & $7074 S$ & goat & & \\
\hline Anti-chicken IgY & AB_2534096 & Alexa Fluor 488 & Thermo Fisher & A11039 & goat & & \\
\hline Anti-guinea Pig & AB_2337438 & $\begin{array}{l}\text { Alexa } \\
\quad \text { Fluor } 488\end{array}$ & $\begin{array}{l}\text { Jackson } \\
\quad \text { ImmunoResearch }\end{array}$ & $106-545-003$ & goat & & \\
\hline Anti-mouse IgG & AB_2338685 & Су3 & $\begin{array}{l}\text { Jackson } \\
\quad \text { ImmunoResearch }\end{array}$ & $115-165-062$ & goat & & \\
\hline Anti-Mouse IgG & AB_2340846 & Alexa Fluor 488 & $\begin{array}{l}\text { Jackson } \\
\quad \text { ImmunoResearch }\end{array}$ & $715-545-150$ & donkey & & \\
\hline $\begin{array}{l}\text { Anti-mouse } \operatorname{lgM}, \mu \\
\text { chain specific }\end{array}$ & AB_2340844 & Alexa Fluor 488 & $\begin{array}{l}\text { Jackson } \\
\text { ImmunoResearch }\end{array}$ & $715-546-020$ & donkey & & \\
\hline Anti-rabbit IgG & AB_2307443 & Суз & $\begin{array}{l}\text { Jackson } \\
\quad \text { ImmunoResearch }\end{array}$ & $711-165-152$ & donkey & & \\
\hline Anti-rabbit IgG & AB_143165 & Alexa Fluor 488 & Thermo Fisher & A11008 & goat & & \\
\hline Anti-rat IgG & AB_2535794 & Alexa Fluor 488 & Thermo Fisher & A-21208 & donkey & & \\
\hline Streptavidin & AB_2337246 & Cy2 & $\begin{array}{l}\text { Jackson } \\
\text { ImmunoResearch }\end{array}$ & 016-220-084 & & & \\
\hline Streptavidin & AB_2337244 & Суз & $\begin{array}{l}\text { Jackson } \\
\text { ImmunoResearch }\end{array}$ & 016-160-084 & & & \\
\hline
\end{tabular}


$\mathrm{mM} \mathrm{Na} \mathrm{Na}_{3}$ GTP-GTP (Devlin et al., 2015). The solution for studying inhibitory synapses was composed of $135 \mathrm{mM}$ Cs-MeSO ${ }_{4}, 10 \mathrm{mM} \mathrm{CsCl}, 10 \mathrm{mM}$ HEPES, $0.5 \mathrm{mM}$ EGTA, and $4 \mathrm{mM} \mathrm{Mg-ATP.} \mathrm{Pipette} \mathrm{solutions} \mathrm{were} \mathrm{freshly} \mathrm{made}$ and filtered $(0.1 \mu \mathrm{m})$, osmolarity was $295-305 \mathrm{mOsmol}$, and pipette resistance was 5-7 M 2 .

To separate IPSCs and EPSCs (typically throughout the entire experiment), different holding potentials were applied $(\mathrm{Vh}=0$ and $\mathrm{Vh}=-60$ or -70 for IPSCs and EPSCs, respectively. All recordings were performed using a MultiClamp 700B amplifier (Molecular Devices). Recordings and pre-processing of data were made with WinWCP (University of Strathclyde). The signals were typically lowpass filtered with a corner frequency $(-3 \mathrm{~dB})$ of $3 \mathrm{kHz}$ and sampled at $6 \mathrm{kHz}$ by DigiData 1322A (Molecular Devices).

Data analysis was performed with p-CLAMP 10 (Molecular Devices) and OriginLab 8 (OriginLab Corp.). sPSCs were detected with a threshold of 5-7 pA, depending on the noise level via template-based algorithm and by visual verification. Analyses of the kinetic properties of SPSCs included only well separated (interevent intervals $>40$ $\mathrm{ms})$, monophasic PSCs which appeared to rise in a monotonic fashion without visible deviation of the rising phase and which decayed exponentially. The rise and decay time courses of individual PSCs were estimated by curve fitting with the exponential function:

$$
A(t)=A_{0} e^{-\frac{t}{\tau}},
$$

where $A(t)$ is the current as a function of time, $A_{0}$ is the maximum amplitude of the event (determined as the maximum current of the selected event/response), $\tau$ is the time constant, $\tau_{\text {RISE }}$ for event's rise phase and $\tau_{\text {DECAY }}$ for decay phase

\section{Differentiation of $\mathrm{mNSCs}$ line}

Primary $\mathrm{mNSC}$ lines isolated from dissected forebrain of E14.5 embryos from Tiparp ${ }^{+/+}$and Tiparp ${ }^{-/}$mice were established and cultured as described previously (Pollard, 2013). In brief, the forebrain was dissected out in dissection solution $(137 \mathrm{mM} \mathrm{NaCl}, 5.4 \mathrm{mM} \mathrm{KCl}, 0.17 \mathrm{mM}$

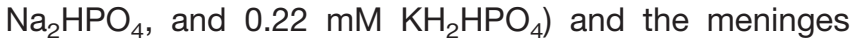
were removed. Each forebrain was kept separately in $1 \mathrm{ml}$ of Accutase and put at $37^{\circ} \mathrm{C}$ for $30 \mathrm{~min}$ and triturated with a pipette half way through the incubation. The cells were then triturated again to obtain an almost a single cell suspension in $1 \mathrm{ml}$ of culture medium [DMEM/F12 supplemented with $1 \%$ penicillin/streptomycin (P/S), 2\% B27, $10 \mathrm{ng} / \mathrm{ml}$ EGF, $10 \mathrm{ng} / \mathrm{ml} \mathrm{FGF}$, and $2.5 \mathrm{U} / \mathrm{ml}$ heparin] and centrifuged $100 \times g$ for $4 \mathrm{~min}$. The pellet was then resuspended in $1 \mathrm{ml}$ of culture medium and the non-digested tissue was left to settle by gravity for about $1 \mathrm{~min}$. Cell suspension was then plated in a well of a 12-well plate. The cells were cultured as a monolayer with laminin. Differentiation of the mNSCs in the three lineages was done as previously described (Conti et al., 2005; Glaser et al., 2007; Pollard, 2013). Briefly, for neuronal differentiation cells were placed in culture medium lacking EGF for a week before changing to complete neurobasal medium (neurobasal medium containing 1\% P/S, 2\% B27, and $1 \%$ GlutaMAX). Experiments were done after a total of $14 \mathrm{~d}$ of differentiation and were maintained in complete neurobasal medium supplemented with $2 \mathrm{ng} / \mathrm{ml}$ BDNF (Peprotech). To differentiate the cells into astrocytes they were placed in DMEM containing $1 \%$ GlutaMAX, $1 \% \mathrm{P} / \mathrm{S}$, and $20 \%$ fetal bovine serum for $7 \mathrm{~d}$ before starting the experiments. Lastly, for oligodendrocyte differentiation cells were cultured for $4 \mathrm{~d}$ in DMEM/F12 containing $1 \%$ $\mathrm{P} / \mathrm{S}, 2 \%$ B27, $10 \mathrm{ng} / \mathrm{ml} \mathrm{FGF,} 2.5 \mathrm{U} / \mathrm{ml}$ heparin, $10 \mathrm{ng} / \mathrm{ml}$ PDGFAA (Peprotech), and $10 \mu \mathrm{M}$ forskolin. This is to support the glial precursor stage. To then induce differentiation, growth factors were withdrawn and cells were exposed to $30 \mu \mathrm{g} / \mathrm{ml} \mathrm{3'3'5-triiodo-L-thyronine} \mathrm{and} 200$ $\mu \mathrm{M}$ ascorbic acid (Sigma) for $4 \mathrm{~d}$. All cell culture reagents were purchased from Thermofisher Scientific, unless stated otherwise.

\section{Cell proliferation and cell death assays}

Cell proliferation was assessed by methylene blue colorimetric assay as previously described (Pelletier et al., 1988). In brief, for each cell line and timepoint cells were seeded in five wells of a 96 -well plate and fixed with $4 \%$ PFA for $15 \mathrm{~min}$ at room temperature at the indicated timepoints. They were then gently washed with PBS and incubated with methylene blue solution ( $1 \% \mathrm{w} / \mathrm{v}$ methylene blue in $0.1 \mathrm{M}$ borate buffer; $\mathrm{pH}$ 9) for $30 \mathrm{~min}$ at room temperature. The excess dye was washed off with four rinses in $0.01 \mathrm{M}$ borate buffer $\mathrm{pH} 9$. The methylene blue was extracted using a 1:1 v/v of ethanol: $0.1 \mathrm{M} \mathrm{HCl}$. The optical density was read at $650 \mathrm{~nm}$ with a Synergy 1 microplate reader.

Cell death was assessed by adding $1 \mu \mathrm{g} / \mathrm{ml}$ Hoechst 33258 to the cell culture media for $1.5 \mathrm{~h}$ and then adding $5 \mu \mathrm{g} / \mathrm{ml}$ propidium iodine and incubating for a further 30 min. The cells were then imaged with a Zeiss Axio Observer microscope and the ratio of live to dead cells calculated with CellProfiler 3.0 software (www.cellprofiler.org; Broad Institute; Carpenter et al., 2006).

Cell migration was assessed by a scratch assay modified from a previous study (Liang et al., 2007). For each cell line and timepoint, five wells of a 24-well plate each containing custom made $1 \times 5 \times 1 \mathrm{~mm}$ silicon inserts. Once the cells were confluent the insert was removed and the cells were placed back into an incubator. At the various timepoints the cells were fixed for $15 \mathrm{~min}$ at room temperature with $4 \%$ PFA and rinsed with PBS. The scratch was imaged with a Zeiss Axio Observer microscope by phase contrast. The images were then analyzed using the Fiji software (https://fiji.sc/; Schindelin et al., 2012).

\section{Cell-cycle analysis}

Cell-cycle analysis was performed by DNA staining of cells followed by flow cytometry. Approximately one million cells per mNSCs line were resuspended by adding $1-\mathrm{ml}$ methanol at $-20^{\circ} \mathrm{C}$ drop by drop while vortexing. Cells were incubated for at least $1 \mathrm{~h}$ at $-20^{\circ} \mathrm{C}$, then $3 \mathrm{ml}$ of PBS were added, and the cells were spun at $200 \times g$ for $3 \mathrm{~min}$. The cell pellet was resuspended in $500 \mathrm{ml}$ of PBS containing $1.5 \mathrm{mg} / \mathrm{ml}$ of Hoechst 33258 . After $30 \mathrm{~min}$, the cells were strained and analyzed by flow ytometry. 


\section{Immunoprecipitation}

Mono-ADP-ribosylation (MAR) of $\alpha$-tubulin was verified by immunoprecipitation as previously described (Hutin et al., 2018a). In brief, mNSCs were harvested and the cell pellet was lysed in cell lysis buffer mNSCs $(200 \mathrm{mM} \mathrm{NaCl}$, $1 \% \mathrm{NP} 40$, and $20 \mathrm{mM}$ HEPES at $\mathrm{pH} 7.4$, supplemented with protease inhibitor cocktail; Sigma). Lysates were clarified by centrifugation and the supernatant was incubated for $2 \mathrm{~h}$ with anti- $\alpha$-tubulin (Sigma) antibody and protein G Dynal beads (Thermo Fisher). The beadantibody-protein complex was then washed three times for $5 \mathrm{~min}$ in wash buffer $(200 \mathrm{mM} \mathrm{NaCl}, 0.1 \% \mathrm{NP} 40$, and $20 \mathrm{mM}$ HEPES at $\mathrm{pH} 7.4$, supplemented with protease inhibitor cocktail; Sigma). The proteins were then eluted from the beads with $2 \times$ Laemmli buffer and separated by SDS-PAGE and transferred to PVDF membranes. Membranes were probed with anti-mono-ADP-ribose-binding reagent (Millipore) followed by incubation with the appropriate secondary antibody. A list of all antibodies is provided in Table 2.

COS- 1 cells were transfected with $2 \mu \mathrm{g}$ of EGFP, GFPTiparp, or GFP-Tiparp ${ }^{\mathrm{H} 532 \mathrm{~A}}$ plasmids (MacPherson et al., 2013) per well of a six-well plate using Lipofectamine 2000 reagent according to the manufacturer's instructions. The next morning, cells were harvested, protein extracts were made and the immunoprecipitation was conducted as described above.

\section{Statistical analyses}

All data were presented as means and SEM. A twotailed Mann-Whitney post hoc, a Kruskal-Wallis one-way ANOVA test followed by a Dunn's multiple comparison, a two-way ANOVA test or Student's $t$ test were used to assess statistical significance $(p<0.05)$. $U$ values for the Mann-Whitney test are reported in the Extended Data Figures 1-1, 4-1, 5-1.

\section{Results}

Tiparp mRNA expression levels were analyzed in the cortex, hippocampus, cerebellum and midbrain of two- to four-month-old C57BL/6N mice, as well as in the liver for comparison. High mRNA levels were observed in the cortex and hippocampus compared with the liver ( $\sim 4$ and 3.8 times higher than in liver, respectively) as well as in the brainstem, which included the diencephalon, and the cerebellum, albeit at lower levels ( 2.5 and 2 times higher than in liver, respectively; Fig. 1A). To establish the role of Tiparp in the brain, we performed histologic analysis on the cerebral cortex of Tiparp $^{-/-}$and Tiparp ${ }^{+/+}$mice at two to four months of age. The distribution of cells along the thickness of the cortex was analyzed by determining the $y$-position of each Nissl-stained cell in comparable sagittal brain sections followed by data binning. Tiparp ${ }^{-/}$mice had a significantly higher percentage of cells in the upper layers of the cortex compared with the lower ones (Fig. $1 B)$. Neuronal-specific NeuN staining suggested that the aberrant distribution of cells along the height of the cortex was due to mislocalization of neurons. Tiparp ${ }^{-1-}$ mice had significantly more NeuN-positive cells in the upper layers at the expense of the lower ones compared with Tiparp $^{+/+}$mice (Fig. 1C). Despite the differences in the cell distribution, they had comparable total cell numbers throughout the thickness of the cortex (Fig. 1B,C, lower panels).

The majority of the differences in cell distribution along the thickness of the cortex were observed around bins 5-15 (Fig. 1A,B). Because of this observation, we then investigated whether this aberrant organization of the neurons along the cortex was due to impaired migration of the neurons from the deep layers to the upper layers during development. Thus, we analyzed the expression of a specific Layer V marker, Ctip2 (Chen et al., 2008). The cortex of Tiparp $^{-/}$mice had significantly less Ctip2positive cells in the middle of the cortex (bins 15-20), but more in the lower third of the cortex compared with Tiparp $^{+/+}$ones (Fig. 1D). To further show disorganization of the cortex we also stained for Satb2, which is expressed in all, but predominantly, layers in II-V (Chen et al., 2008; Huang et al., 2013). The cortex of Tiparp ${ }^{+/+}$ mice had more Satb2-positive neurons in bins 15-35 and significantly less in bins 35-40 compared with Tiparp ${ }^{-/}$ mice (Fig. 1E). Taken together, these data suggest that the loss of Tiparp causes aberrant positioning of various neuronal subtypes across all layers and the entire thickness.

As TCDD has been previously shown to affect GABAergic neurons and TCDD increases Tiparp expression via Ahr, we investigated whether loss of Tiparp had any effects on GABAergic neurons and their distribution. The majority of these neurons in the cortex can be divided into subtypes based on the differential expression of calcium binding proteins: calbindin, calretinin or parvalbumin (Condé et al., 1994; del Río and DeFelipe, 1996; Gabbott and Bacon, 1996). Sagittal brain sections from Tiparp ${ }^{+/+}$ and Tiparp $^{-1-}$ mice were stained for the three calcium binding proteins. Tiparp ${ }^{-/-}$mice had significantly lower (29\%) calretinin and lower (40\%) parvalbumin-positive neurons, but higher calbindin (148\%) expressing cells compared with Tiparp $^{+/+}$littermates (Fig. $2 A, C$ ). Perineuronal nets (PNNs) are extracellular matrix structures that preferentially surround parvalbumin expressing GABAergic neurons (Kosaka and Heizmann, 1989; Härtig et al., 1992). We then investigated if the decrease in parvalbumin expressing neurons correlated with a decreased formation of PNNs. Tiparp ${ }^{-1}$ mice had lower number of cells $(49 \%)$ surrounded by these structures (Fig. 2B,C). However, whether loss of PNNs was due to a direct effect of Tiparp on the extracellular structures or whether this was due to a decrease in parvalbumin-positive neurons remains to be studied. Taken together, these data suggest that Tiparp affects the number and correct distribution of GABAergic neurons in the cortex.

To determine whether Tiparp loss impaired neuronal networks and interfered with the excitability of cortical pyramidal neurons and the alternation of cortical microcircuitry, we did whole-cell patch-clamp recordings. Specifically, we investigated the characteristic current-voltage (I-V) curves from action potentials to assess the firing rate of pyramidal neurons and assessed the minimal current injection required to trigger enough membrane depolarization for the cells to generate an action potential. Both EPSCs and 
A

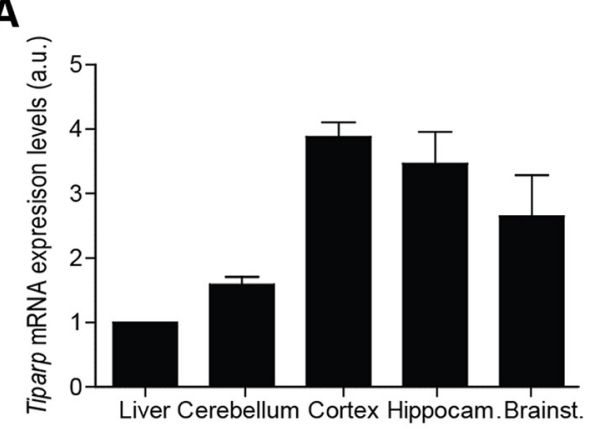

B

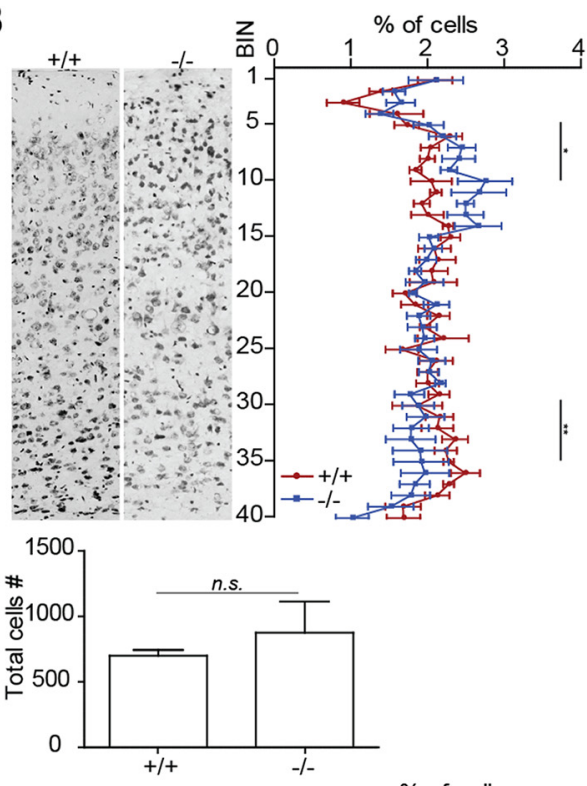

D
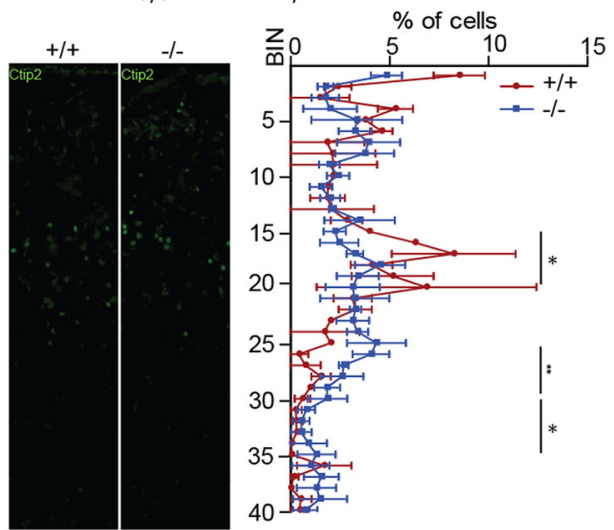

C
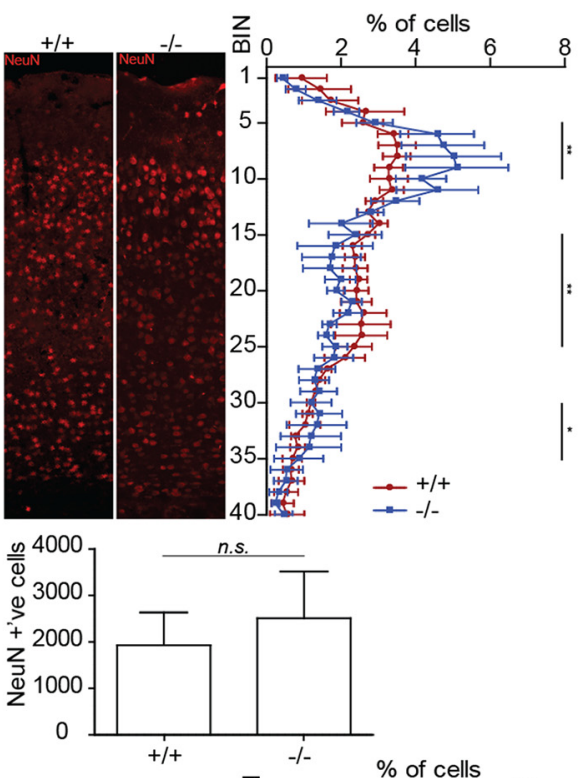

E
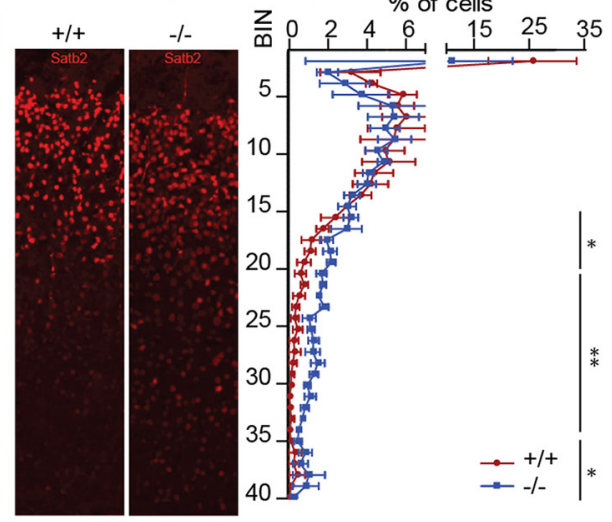

Figure 1. Tiparp is highly expressed in the adult mouse brain and its depletion leads to aberrant layering. $\boldsymbol{A}$, qPCR of Tiparp gene expression levels in cortex, cerebellum, hippocampus (hippocam.) and brainstem (brainst.), which also included the diencephalon relative to liver $(n=3)$. $\boldsymbol{B}-\boldsymbol{E}$, Representative stainings of comparable sagittal sections from Tiparp ${ }^{+/+}$and Tiparp $^{-/-}$mouse cortex followed by automated counting of cells and distribution analysis along the $y$-axis of the cortex. Graphs below $\boldsymbol{B}, \boldsymbol{C}$ show the total number of cells $(\boldsymbol{B})$ and NeuN-positive cells $(\boldsymbol{C})$ in the area analyzed. $\boldsymbol{B}$, Nissl staining in adult mouse $(n=5)$. $\boldsymbol{C}$, Neurons stained with NeuN in the adult mouse $(n=3)$. $\boldsymbol{D}$, Ctip2 staining of neurons in Layer V in P5 mouse $(n=3)$. $\boldsymbol{E}$, Satb2 staining predominantly of neurons in Layers II-V in P5 mouse $(n=3)$. Percentage of cells refers to the percentage of cells in a bin relative to the total number of cells in the area analyzed. The asterisk denotes significant differences between the wild type and the knock-out $(* p<0.05$ or $* * p<0.005)$, within the delimited bin range, according to a two-tailed Mann-Whitney test. $U$ values for the Mann-Whitney test can be found in Extended Data Figure 1-1. Non-significance is denoted with n.s. Data are mean \pm SEM. The $n$ number refers to the number of mice in each group, for panels $B-E$, multiple sections of each mouse brain were analyzed, but statistics was done per animal. 
$\mathbf{A}$
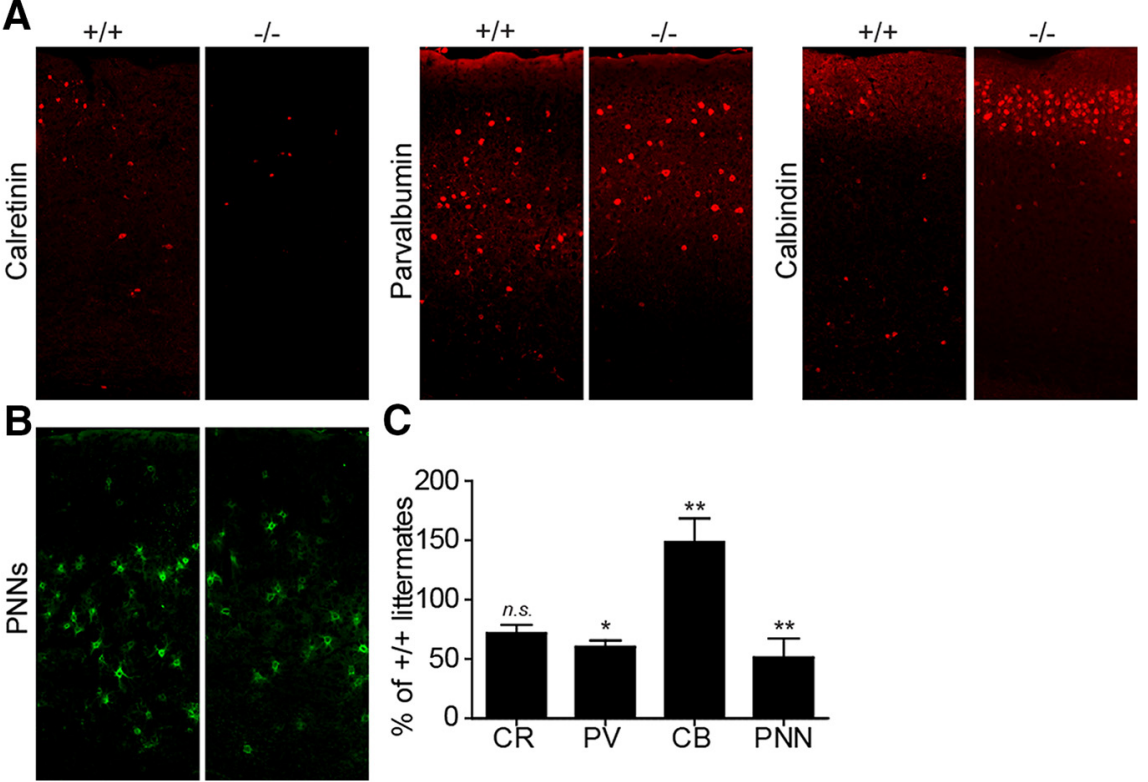

Figure 2. Tiparp $^{-/-}$mouse cortex presents altered number of GABAergic neurons. $\boldsymbol{A}$, Fluorescent micrographs from representative staining for calretinin (CR), parvalbumin (PV), calbindin (CB), or $\boldsymbol{B}$, perineuronal nets (PNNs) in comparable sagittal sections from Tiparp ${ }^{+/+}$and Tiparp ${ }^{-/-}$adult mouse cortex. The green fluorescent staining of the PNNs was obtained by biotinylated WFA and Cy2-streptavidin. C, Quantification of calretinin, parvalbumin, calbindin expressing cells, and cells surrounded by PNNs, in Tiparp $^{-1}$ adult mouse cortex, expressed as percentage of the wild-type littermate $(n=3$ mice). Data are mean \pm SEM. The asterisk denotes significant differences between the wild type and the knock-out $(* p<0.05$ or $* * p<0.005)$ calculated with a two-way ANOVA test. Non-significance is denoted with n.s.

IPSCs were recorded. However, no significant differences were observed in any of these parameters between neurons isolated from Tiparp $^{-/-}$and Tiparp ${ }^{+/+}$mice (Fig. $3 A-F$ ). The resting potential of the cells was also measured and it showed hyperpolarization of Tiparp ${ }^{-1}$ neurons compared with those from Tiparp ${ }^{+/+}$mice (Fig. 3F), suggesting alterations in function and/or number of ion channels.

To elucidate the developmental mechanisms underlying the aberrant distribution of neurons in the cortex, and to understand whether the observed phenotype was due to aberrant differentiation, proliferation or migration of developing neurons, we isolated mNSCs from both Tiparp $^{+/+}$and Tiparp ${ }^{-/}$littermates. Three independent lines were established from two litters. There were no significant differences in the expression of the basic NSC markers at either mRNA level (SOX2, PAX6, Nestin) between genotypes. mNSCs derived from both genotypes expressed comparable protein levels of SOX2 and Nestin (Fig. 4A). Both Tiparp ${ }^{+/+}$and Tiparp ${ }^{-/-}$mNSCs differentiated into the three neural lineages: neurons, astrocytes and oligodendrocytes. Differentiation into neurons resulted in increased RNA expression levels of the neuronal markers Tubb3, Nfh, and Dcx compared with mNSCs. However, no significant differences in these markers were observed between genotypes (Fig. 4B). Astrocyte differentiation resulted in increased RNA expression levels of astrocyte-specific markers compared with undifferentiated cells (Fig. 4C). Of the three markers examined, we only observed significant increases in Blbp RNA levels between Tiparp $^{--}$and Tiparp ${ }^{+/+}$cells. For oligodendrocyte differentiation, significant increases in Pdgfra and
Ng2 were observed in both genotypes compared with undifferentiated cells. The oligodendrocyte marker, $\mathrm{Ng} 2$, was significantly increased at the RNA level in Tiparp ${ }^{-/}$ compared with Tiparp ${ }^{+/+}$cells (Fig. 4D). Olig1 RNA levels were significantly lower in Tiparp ${ }^{-/-}$compared with Tiparp $^{+/+}$mNSCs. Olig1 levels were significantly decreased in differentiation to oligodendrocytes in Tiparp ${ }^{+/+}$ cells, but unchanged in Tiparp $^{-/-}$cells. At protein level, Tiparp $^{-/-}$mNSCs differentiated into the three lineages express the typical markers, suggesting that even in the absence of Tiparp the cells retain their differentiation potential (Fig. 4B-D). Tiparp mRNA levels were significantly downregulated during neuronal cell differentiation, whereas they were significantly upregulated on astrocyte differentiation (Fig. 4E). No changes were observed when cells were differentiated toward the oligodendrocyte lineage (Fig. 4E). This suggested that Tiparp expression is regulated during neuronal and astrocytic differentiation and it is involved in the regulation of specific genes involved in differentiation, but it is not a master regulator of neuronal stem cell differentiation.

Next, we assessed the proliferation rate of Tiparp ${ }^{+/+}$ and Tiparp ${ }^{-1-}$ mNSCs by conducting a methylene blue assay. Indeed, despite having plated the same number of cells after $72 \mathrm{~h}$, Tiparp $^{-/-}$mNSCs had proliferated significantly less than the Tiparp ${ }^{+/+}$cells (Fig. 5A). Since no differences in propidium iodine staining were observed between the two genotypes, increases in cell death could not be explained by the slower growth rate of the Tiparp ${ }^{-/}$ cells (Fig. 5B). We then stained the cells with Hoechst 33342 and subjected them to flow cytometry to determine the percentage of cells in each phase of the cell-cycle cell. 
A

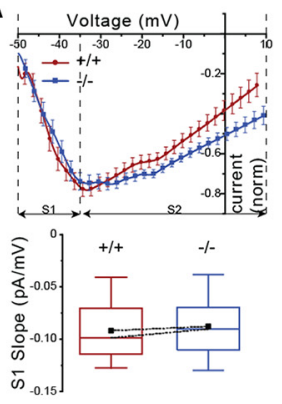

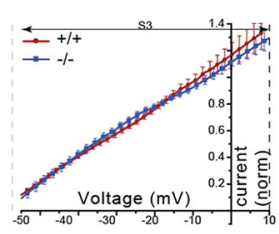

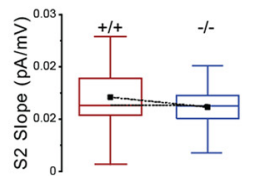

$\mathbf{F}$
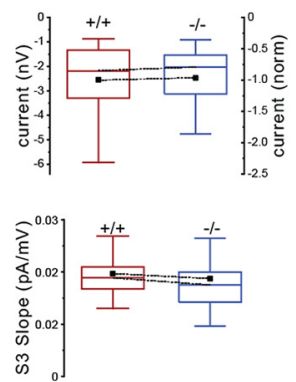
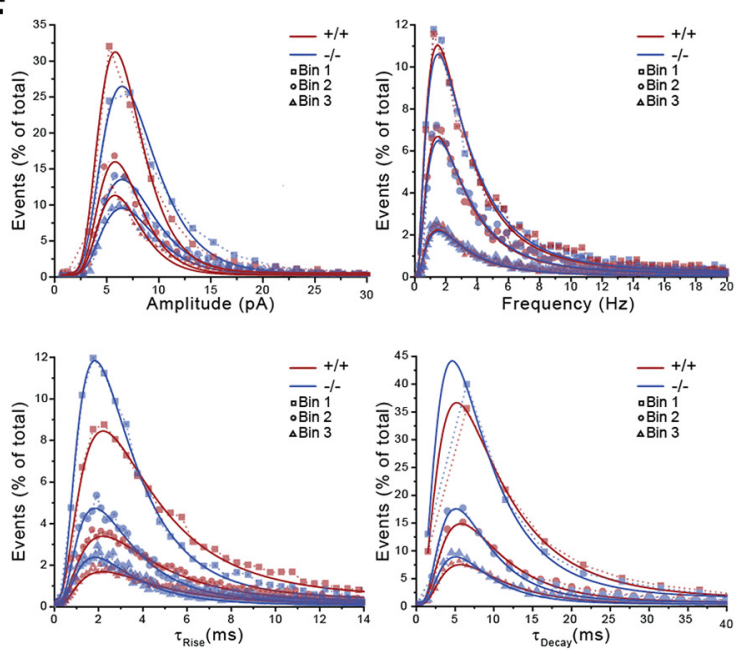

$\mathbf{E}$
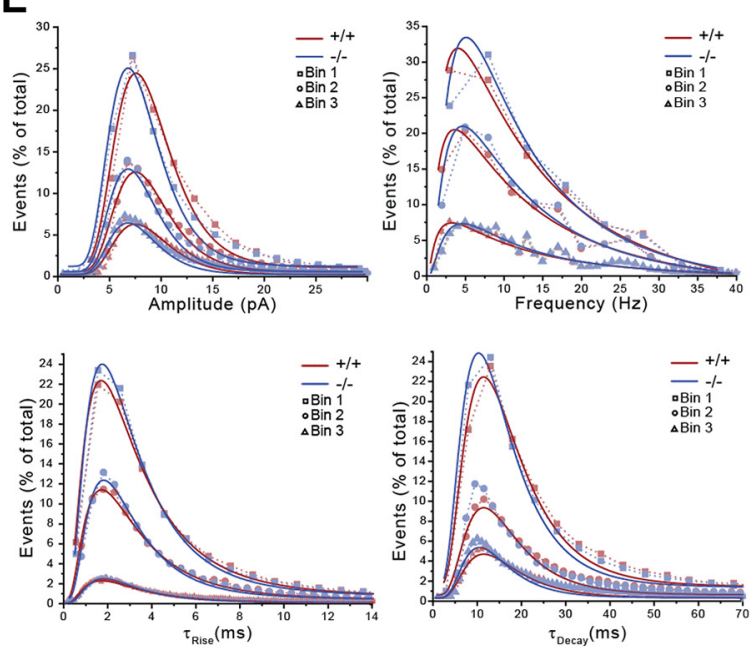

Figure 3. Knock-out of Tiparp does not affect the electrophysiology of cortex primary neurons. $\boldsymbol{A}$, Characterization of the currents from voltage-clamp recordings show comparable potential dependence of induced currents in the two genotypes. Averaged current-voltage curves (I-V) of inward currents (top left panel) and outward currents (top middle panel) for voltage clamp recordings from whole cells in Tiparp ${ }^{+/+}$and Tiparp ${ }^{-/}$adult mouse cortex slices. The top right panel is a box chart representation of the maximum amplitudes of the inward currents. The bottom panel shows box chart representations of the averaged slopes of corresponded periods of the individual IV curves: the inward currents (S1 for the rise and S2 for the decay period; left and middle panel, respectively) and the entire outward currents (slope S3; right panel). These are all calculated for each recording independently. The box represents the data between the second and third quartile, the whiskers represent the range within the 1.5 interquartile range. The black square is the mean and the line within the box is the median value. Current (norm.) is the normalized current expressed in arbitrary units. B. Frequencies of action potential firing during step membrane depolarization are similar for Tiparp ${ }^{+/+}$and Tiparp $/-$ neurons. Concatenated curves (left panel) and the averaged curves of the individual cells (right panel) for depolarization dependent frequencies of action potential firing of Tiparp ${ }^{+/+}$and Tiparp ${ }^{-/-}$neurons. The allometric function fittings $f(x)=a * x^{\wedge} b$, where $a$ and $b$ are parameters, were also plotted on the graphs. $\boldsymbol{C}$, Minimal current injection required to trigger enough membrane depolarization for the cells to generate an action potential firing. Distribution curves and histograms of concatenated data of current injections in Tiparp $^{+/+}$and Tiparp $^{-/-}$cells needed for action potential (AP) generation calculated for bin $=10 \mathrm{pA}$ (left panel) and bin $=20$ pA (middle panel) step size. The $y$-axis represents the fraction of cells depolarized by a given current injection ( $x$-axis) sufficient for AP firing. Most of cells start AP firing when depolarized by a $40-$ to $80-p A$ current injection. The right panel is a box plot of the concatenated data that a cell generates an AP under the given minimal current injection. The data does not show normal distribution. $\boldsymbol{D}$, The left panel shows the distribution curves for concatenated data of the resting potential of the patched cells and expressed as the probability to observe a defined resting potential. The graphs represent the Gaussian curves for the data grouped by three different bin sizes. The right panel shows a box chart of the concatenated data of resting potential within the entire data range, the whiskers represent the range within the 1.5 interquartile range. The black square is the mean and the line within the box is the median value. $\boldsymbol{E}, \boldsymbol{F}$, Similarity of functional composition and excitability of Tiparp ${ }^{+/+}$and Tiparp ${ }^{-/}$cortical networks: sPSCs. Whole-cell voltage clamp recordings of sIPSCs $(\boldsymbol{E})$ and SEPSCs $(\boldsymbol{F})$ from Tiparp ${ }^{+/+}$and Tiparp ${ }^{-/-}$adult mouse cortex slices. Concatenated data of the distribution curves for the amplitudes (top left panel); frequencies (top right panel); tau rises (bottom left panel) and tau decays (bottom right panel). The data were grouped into three bins and fitted with lognormal curves. 

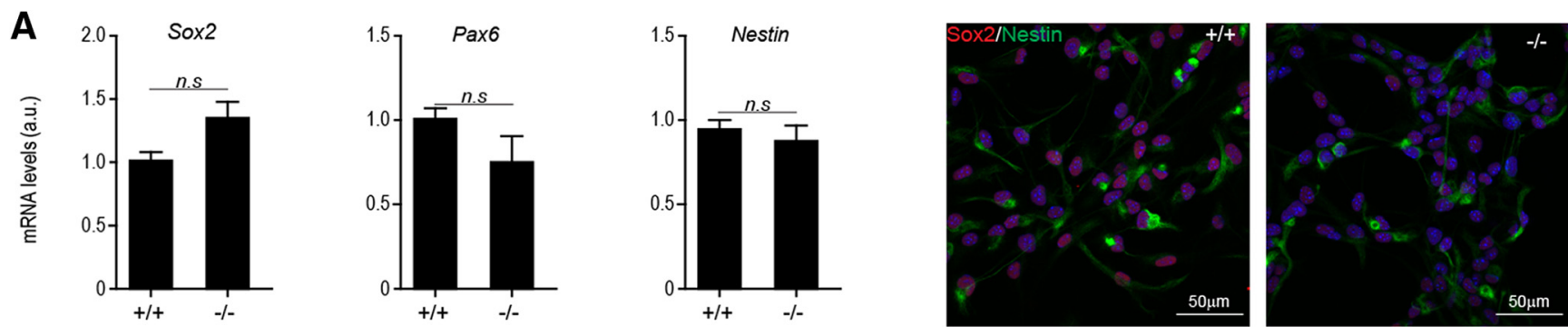

B
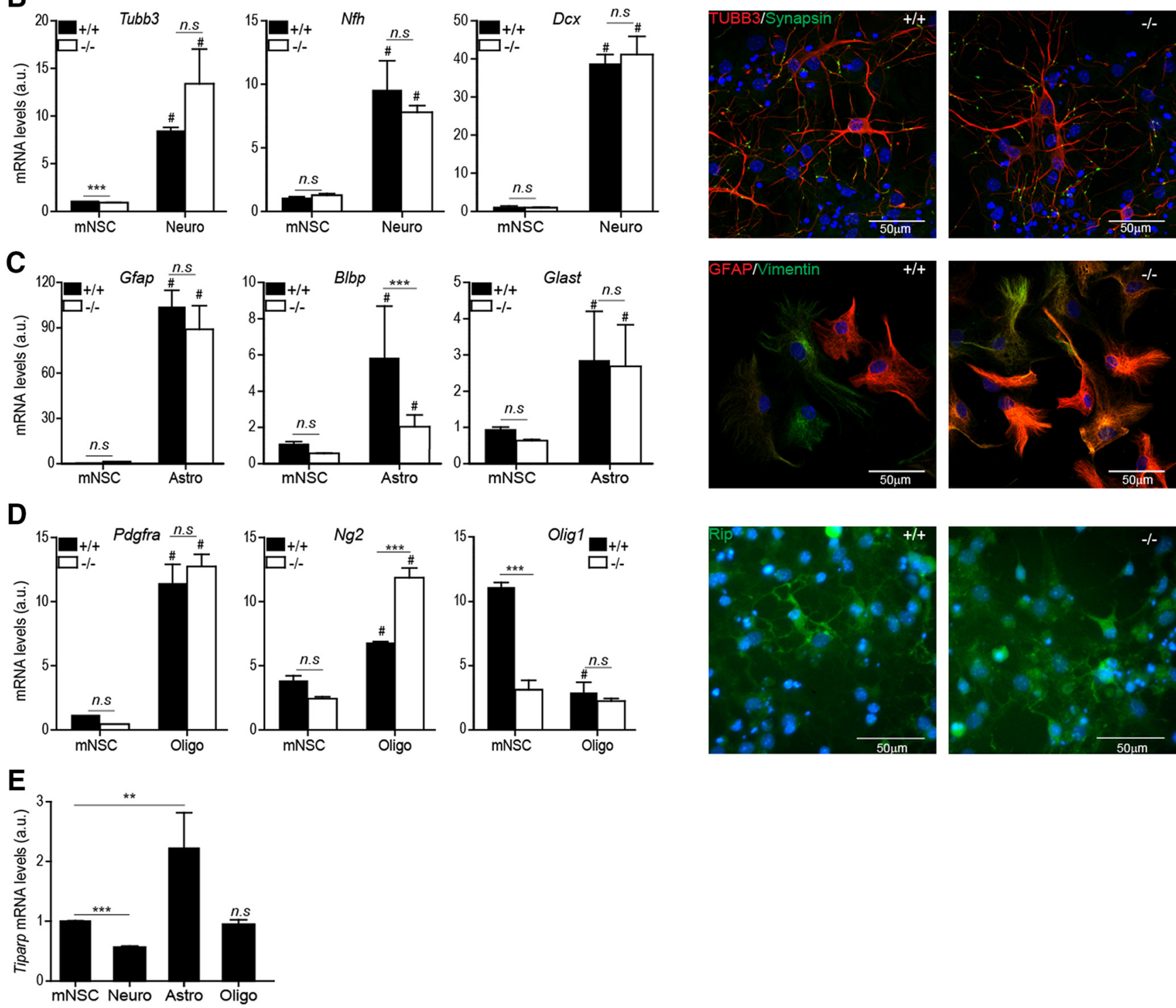

Figure 4. Differentiation potential of Tiparp $^{-/-}$mNSCs into three lineages. $\boldsymbol{A}-\boldsymbol{D}$, Gene (left panel) and protein (right panel) expression of mNSCs markers in undifferentiated cells $(\boldsymbol{A})$; neuronal markers in cells differentiated toward the neuronal lineage (Neuro) for $14 \mathrm{~d}$ $(B)$; astrocyte markers in 7-d differentiated astrocytes (Astro; $\boldsymbol{C}$ ) and oligodendrocyte markers in cells differentiated for $8 \mathrm{~d}$ toward the oligodendrocytic lineage (Oligo; $\boldsymbol{D})$. $\boldsymbol{E}$, Tiparp gene expression levels in Tiparp ${ }^{+/+} \mathrm{mNSCs}$ and in cells differentiated toward the three neural lineages, as $(* * * p<0.001$ or $* * p<0.005)$ between the genotypes determined by a two-tailed Mann-Whitney test $(\mathrm{A}$, $\mathrm{E}$; $\mathrm{U}$ values can be found in Extended Data Fig. 4-1) or a two-way ANOVA (B-D). Non-significance is denoted by n.s., and \# represents statistical significance (at least $p<0.05$ ) between undifferentiated and differentiated cells $(\boldsymbol{B}-\boldsymbol{D})$.

The cycle analysis revealed a significantly lower percentage of Tiparp $^{-/-}$cells in the S-phase, which was accompanied by a slightly higher percentage of cells in $G_{0} / G_{1}$ and $\mathrm{G}_{2} / \mathrm{M}$ phases compared with Tiparp $^{+/+}$cells (Fig. 5 C). To verify this further, mNSCs in mitosis were analyzed in vivo in comparable coronal sections of the prefrontal cor- 

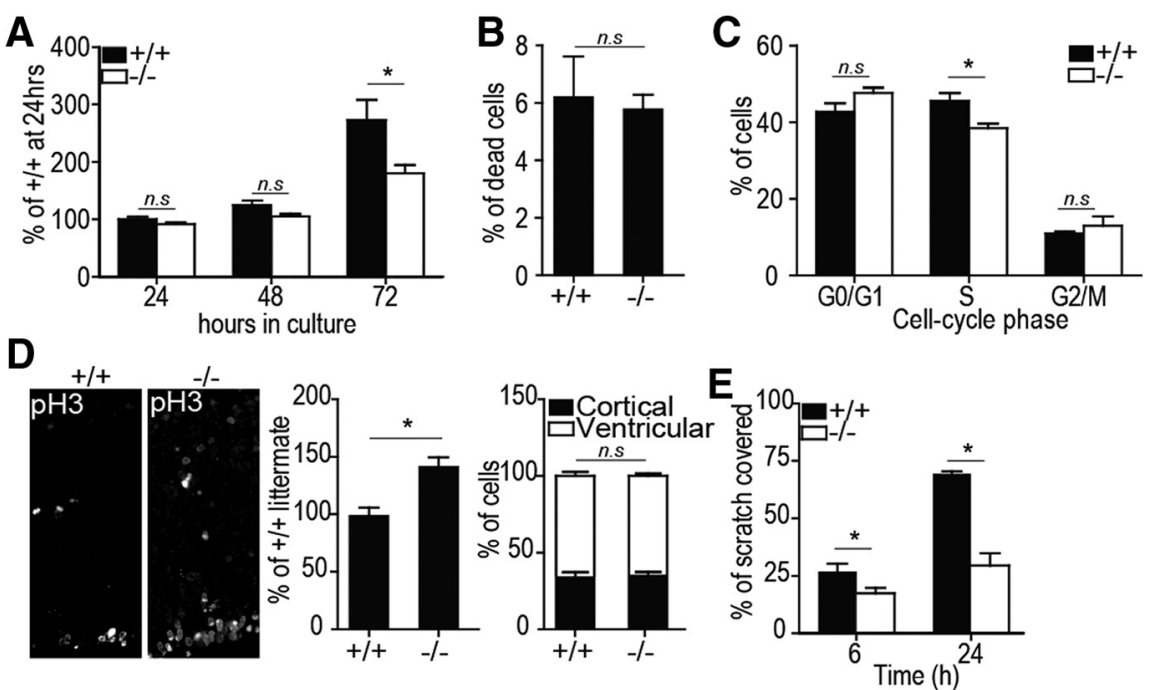

Figure 5. Tiparp depletion affects proliferation and migration of mNSCs. A, Methylene blue assay of Tiparp ${ }^{+/+}$and Tiparp $^{-/-}$mNSCs to determine proliferation rates expressed as percentage of Tiparp ${ }^{+/+}$cells $24 \mathrm{~h}$ after plating. $\boldsymbol{B}$, Propidium iodine and Hoescht 33342 staining of cells in both Tiparp ${ }^{+/+}$and Tiparp ${ }^{-/-}$mNSCs was used to assess cell death. The graph shows the percentage of propidium iodine-positive cells within each genotype. C. Tiparp ${ }^{+/+}$and Tiparp ${ }^{-/}$mNSCs were stained with Hoescht 33342 and subjected to flow cytometry to determine the percentage of cells in each phase of the cell cycle. All experiments were conducted on three independent mNSCs lines. $\boldsymbol{D}$, Representative image of coronal section of E17.5 Tiparp $^{+/+}$and Tiparp ${ }^{-/}$mice stained with an anti-phosphorylated histone $\mathrm{H} 3$ antibody $(\mathrm{pH} 3)$, left panel and quantification of the $\mathrm{pH} 3$-positive cells as percentage of the wild-type littermate (middle panel) and percentage of total cells in each of the two pH3-positive cell populations determined by their position in the cortical plate or in the ventricular zone (right panel; $n=3$ mice). $\boldsymbol{E}$. Scratch assay to assess migration capabilities of Tiparp ${ }^{+/+}$and Tiparp $^{-/-}$ mNSCs. Data are mean \pm SEM. The asterisk denotes significant differences between the wild type and the knock-out $(p<0.05)$ with a two-way ANOVA $(\boldsymbol{A}, \boldsymbol{C}, \boldsymbol{D}$, right panel, $\boldsymbol{E})$ or a Mann-Whitney test $(\boldsymbol{B}, \boldsymbol{D}$, middle; $U$ values can be found in Extended Data Fig. 5-1). Non-significance is denoted by n.s.

tex from Tiparp ${ }^{+/+}$or Tiparp ${ }^{-/}$E13.5 mice stained with an anti-phosphorylated histone $\mathrm{H} 3$ antibody (serine 10). Histone $\mathrm{H} 3$ is specifically phosphorylated during mitosis where metaphase chromosomes are phosphorylated and on exit of the cell cycle a global dephosphorylation of $\mathrm{H} 3$ occurs (Hans and Dimitrov, 2001). As expected, prefrontal cortex in Tiparp ${ }^{-/-}$mice had significantly higher levels of phospho-histone H3-positive cells compared with their Tiparp $^{+/+}$littermates. Interestingly, both the ventricular and aventricular mNSC populations were increased in the Tiparp $^{-/}$mice, but the ratio of the two populations was the same in both genotypes (Fig. 5D). Taken together, these data suggest a mitotic defect with abnormal cellcycle progression resulting in a lower proliferation rate in the absence of Tiparp. Furthermore, our findings are consistent with a previous report showing that TIPARP knock-down in HeLa cells results in higher phosphohistone $\mathrm{H} 3$ staining and an increased length of premetaphase mitosis (Vyas et al., 2013).

In vitro migration assays of NSCs can be an indication of whether migration could also be affected in vivo. Thus, we assessed the effect of Tiparp loss on mNSC migration capabilities with a scratch assay. Six hours after the scratch through the center of confluent cells, Tiparp ${ }^{+/+}$ mNSCs covered $\sim 28 \%$ of the scratch versus only $18 \%$ covered by the Tiparp $^{-/-}$cells. This difference increased after $24 \mathrm{~h}$, by which time, Tiparp $^{-1-}$ cells covered $31 \%$ of the scratched area while the Tiparp ${ }^{+/+}$mNSCs covered $73 \%$ (Fig. 5E). Although the molecular mechanisms underlying the aberrant distribution of neurons in the cortex remain unknown, our data suggest that cell cycle/proliferation and cell migration are two processes affected by the absence of Tiparp which could contribute to the observed phenotype.

Since previous studies reported a link between $\alpha$-tubulin and abnormal migration of cortical neurons (Keays et al., 2007; Li et al., 2012) and $\alpha$-tubulin was recently reported to be ADP-ribosylated (Larsen et al., 2018), we explored the possibility that the defects in correct cell layering in the cortex of Tiparp $^{-1}$ mice were due to altered regulation of $\alpha$-tubulin which in turn led to abnormal migration. Western blot analysis showed a significant decrease in the levels of MAR of immunoprecipitated $\alpha$-tubulin in Tiparp ${ }^{-/}$cells compared with Tiparp ${ }^{+/+}$ cells (Fig. 6A). We next transfected COS-1 cells with GFP-Tiparp or a catalytically deficient TIPARP, GFPTiparp ${ }^{\mathrm{H} 532 \mathrm{~A}}$, and repeated the $\alpha$-tubulin immunoprecipitation to verify that it the MAR was Tiparp-dependent. Transfection of GFP-Tiparp in COS-1 cells followed by $\alpha$-tubulin immunoprecipitation showed an increase in MAR compared to cells transfected with GFP alone or with the GFP-Tiparp ${ }^{\text {H532A }}$ (Fig. 6B). The different levels of protein expression detected between wild-type Tiparp and the Tiparp ${ }^{\mathrm{H} 532 \mathrm{~A}}$ catalytic mutant was due to the increased stability of the latter (MacPherson et al., 2013). To show co-localization of Tiparp with tubulin GFP, GFPTiparp and GFP-Tiparp ${ }^{\mathrm{H} 532 \mathrm{~A}}$ were transfected in COS-1 cells and then immunostained with $\alpha$-tubulin. Colocalization was observed in the cells bearing the GFP-Tiparp plasmid, but this was independent of Tiparp catalytic 
A

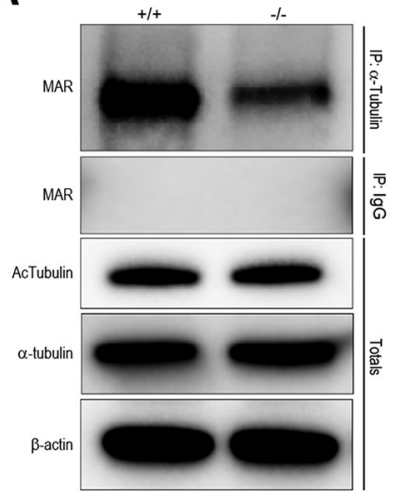

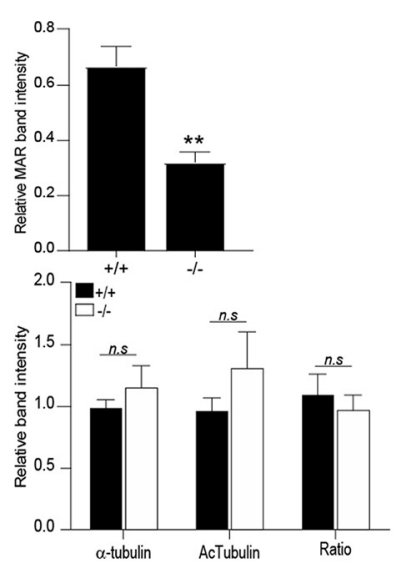

B

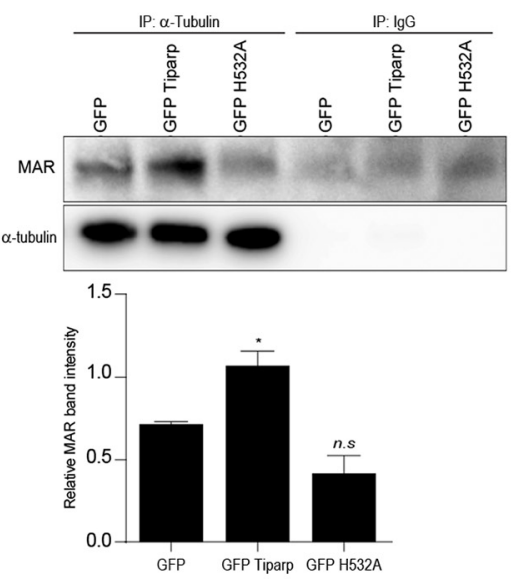

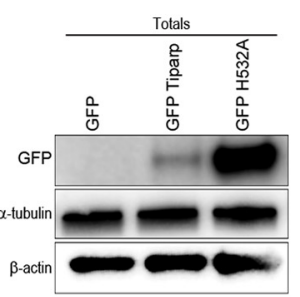

C
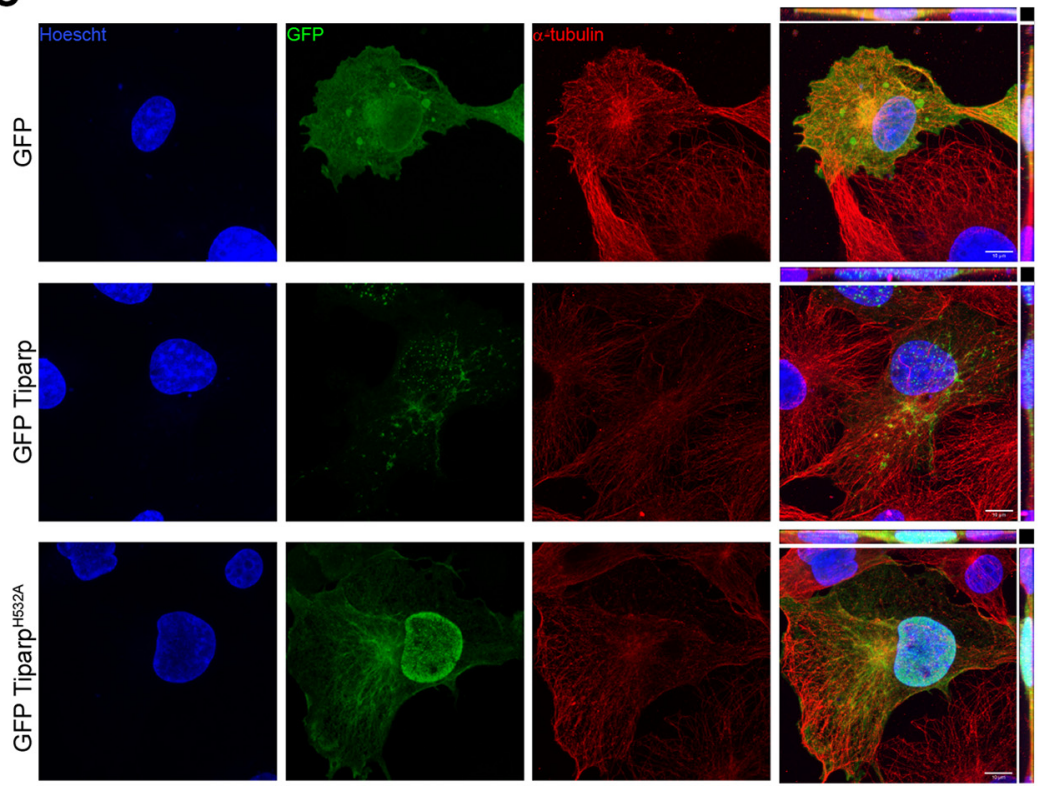

Figure 6. Tiparp ADP ribosylates $\alpha$-tubulin. A, Protein extracts from Tiparp ${ }^{+/+}$and Tiparp ${ }^{-/-}$mNSCs immunoprecipitation of $\alpha$-tubulin followed by western blot against MAR. Total protein extracts were also immunoblotted against acetylated $\alpha$-tubulin and $\alpha$-tubulin. The right panel shows densitometry analysis of the relative intensity of the MAR band normalized to $\beta$-actin (top graph) and relative intensity of the acetylated $\alpha$-tubulin, $\alpha$-tubulin in the totals normalized to $\beta$-actin as well as the acetylated $\alpha$-tubulin to $\alpha$-tubulin ratio (ratio; bottom panel). B, Cell extracts from COS-1 cells overexpressing GFP, GFP-Tiparp or the catalytic mutant GFP-Tiparp ${ }^{\text {H532A }}$ (labeled GFP-TiparpH532A) were immunoprecipitated with $\alpha$-tubulin and immunoblotted against MAR and GFP to show coprecipitation of $\alpha$-tubulin and Tiparp. C, Cos-1 cells overexpressing GFP, GFP-Tiparp, or GFP-Tiparp ${ }^{\mathrm{H} 532 \mathrm{~A}}$ and stained for $\alpha$-tubulin. Data are mean \pm SEM. The asterisk denotes significant differences between the wild type and the knock-out $(* p<0.05$ and $* * p<$ $0.005)$ calculated with an unpaired $t$ test $(\boldsymbol{A}$, top panel), a two-way ANOVA ( $\boldsymbol{A}$, bottom panel), or an ordinary one-way ANOVA (B). Non-significance is denoted by n.s.

activity, since colocalization between $\alpha$-tubulin and GFPTiparp ${ }^{\mathrm{H} 532 \mathrm{~A}}$ was also observed (Fig. 6C). Taken together, these data suggest that Tiparp plays an important role to MAR of $\alpha$-tubulin.

\section{Discussion}

In this study, we demonstrate that Tiparp affects neural progenitor cell proliferation and migration, and its loss leads to aberrant organization of the mouse cortex. Although mild, the phenotype of the Tiparp ${ }^{-/}$mice suggests that alterations in Tiparp expression or function could increase susceptibility to a wide range of both developmental and degenerative neurologic diseases.
We show that Tiparp ${ }^{-/-}$mice have less PNNs, which are structures responsible for synaptic stabilization. This observation agrees with a study which used LTP in rats as a cellular model of learning and memory that identified Tiparp as a highly upregulated gene and essential for the maintenance long-lasting LTP, suggesting a close association with plastic changes in the synaptic efficacy. This is achieved by the induction of structural alteration in the synaptic connections and their deep restructuring (Matsuo et al., 2000). However, whether this loss of PNNs is due to the loss of Tiparp is unknown but it does also suggest aberrant synaptic plasticity. 
Furthermore, adult Tiparp ${ }^{-/}$mice exhibited atypical laminar organization of the cortex with increased number of cells, and specifically neurons, in the upper layers of the cortex. Reduced staining for specific cortical layer markers was especially evident in Layer V of Tiparp ${ }^{-1-}$ mice brains. Differentiation and migration of neuronal progenitors during the development of the neocortex is temporally controlled with the migration of cells to specific laminar layers occurring in an inside-out manner. Our findings suggest that Tiparp influences neuronal cell-class determination (layer destination of projection neurons) through regulation of the cell cycle and migration. Furthermore, the Tiparp ${ }^{-/-}$mice showed differences of GABAergic neuron distribution with lower amounts of calretinin and parvalbumin-positive interneurons, but higher numbers of calbindin-expressing neurons. The molecular mechanisms underlining this observation remain to be determined.

Ahr influences the migration and differentiation of neurons and specifically of GABAergic neurons (Huang et al., 2004; Qin and Powell-Coffman, 2004). Other studies report that activating the Ahr pathway in mice alters differentiation patterns of neural progenitors and leads to decreased numbers of non-GABAergic projection neurons and thinner deep neocortical layers due to the nuclear accumulation of cyclin-dependent kinase inhibitor p27 Kip1 (Kolluri et al., 1999; Mitsuhashi et al., 2010). Moreover, the absence of Ahr expression compromises GABAergic neuron cell differentiation in the ventral telencephalon (Huang et al., 2004; Gohlke et al., 2009). Tiparp expression is induced by Ahr, which in turn regulates Ahr signaling as part of a negative feedback loop (MacPherson et al., 2013; Grimaldi et al., 2018). Therefore, loss of Tiparp could result in hyperactivation of Ahr signaling, which could partly explain our findings. Alternatively, Tiparp may function in the brain on activation of the Ahr pathway and subsequently target critical proteins in different pathways thus acting as an important downstream regulator of Ahr. Tiparp has been described as both a modulator and cross-pathway mediator of innate immunity. Constitutive Ahr signaling targets Tiparp which in turn mono-ADP ribosylates TBK1, thus suppressing the TBK1-mediated pathway that is essential for interferon induction and the innate immune response (Yamada et al., 2016).

However, Tiparp could also function independently of Ahr. Its expression can be induced by other nuclear receptors important in neuronal development such as glucocorticoid receptor and platelet-derived growth factor receptor (Schmahl et al., 2008; Chinenov et al., 2014). Indeed, in utero exposure to synthetic glucocorticoids results in aberrant distribution of neurons throughout the cortex, similar to what we report in this study (Tsiarli, 2014). The complex cytoarchitecture and laminar organization of the cerebral cortex are the result of several processes including neurogenesis, neuronal migration and differentiation. Cortical malformations often show abnormalities in all three processes, because genes involved in one of these processes often affect the other two (Manzini and Walsh, 2011). Cytoskeletal components, such as $\alpha$-tubulin are key regulators of all aspects of neuronal differentiation have been associated with cortical malformations (Francis et al., 2006; Kerjan and Gleeson, 2007; Lian and Sheen, 2015). Tuba1a mutant mice have defects associated with cortical Layers II/III and IV and aberrant neuronal migration (Keays et al., 2007). A recent study reported that $\alpha$-tubulin is monoADP ribosylated; however, the enzyme responsible for this posttranslational modification of $\alpha$-tubulin was not reported (Larsen et al., 2018). Here, we provide evidence that Tiparp contributes to the MAR $\alpha$-tubulin. Loss of Tiparp expression or its catalytically activity resulted in a significant decrease in mono-ADP-ribosylated $\alpha$-tubulin. Since tubulin is regulated by many other mechanisms and other posttranslational modifications, the defects we observed were not as severe as those described in the Tuba1a mutant mice (Keays et al., 2007; Kumar et al., 2010; Magiera and Janke, 2014). Collectively, our results suggest that Tiparp plays a role in the development of the cerebral cortex. Further studies are needed to fully characterize its mechanism of action and to dissect the pathways involved in this regulation.

\section{References}

Ahmed S, Bott D, Gomez A, Tamblyn L, Rasheed A, Cho T, MacPherson L, Sugamori KS, Yang Y, Grant DM, Cummins CL, Matthews $J$ (2015) Loss of the mono-ADP-ribosyltransferase, Tiparp, increases sensitivity to dioxin-induced steatohepatitis and lethality. J Biol Chem 290:16824-16840.

Berthold MR, Cebron N, Dill F, Gabriel TR, Kotter T, Meinl T, Ohl P, Thiel K, Wiswedel B (2009) KNIME - the Konstanz information miner: version 2.0 and beyond. J SIGKDD Explor NewsI 11:26-31.

Carpenter AE, Jones TR, Lamprecht MR, Clarke C, Kang IH, Friman O, Guertin DA, Chang JH, Lindquist RA, Moffat J, Golland P, Sabatini DM (2006) CellProfiler: image analysis software for identifying and quantifying cell phenotypes. Genome Biol 7:R100.

Chen B, Wang SS, Hattox AM, Rayburn H, Nelson SB, McConnell SK (2008) The Fezf2-Ctip2 genetic pathway regulates the fate choice of subcortical projection neurons in the developing cerebral cortex. Proc Natl Acad Sci USA 105:11382-11387.

Chen Y, Sha R, Xu L, Xia Y, Liu Y, Li X, Xie HQ, Tang N, Zhao B (2019) 2,3,7,8-Tetrachlorodibenzo-p-dioxin promotes migration ability of primary cultured rat astrocytes via aryl hydrocarbon receptor. J Environ Sci (China) 76:368-376.

Chinenov Y, Coppo M, Gupte R, Sacta MA, Rogatsky I (2014) Glucocorticoid receptor coordinates transcription factordominated regulatory network in macrophages. BMC Genomics 15:656.

Collins LL, Williamson MA, Thompson BD, Dever DP, Gasiewicz TA, Opanashuk LA (2008) 2,3,7,8-Tetracholorodibenzo-p-dioxin exposure disrupts granule neuron precursor maturation in the developing mouse cerebellum. Toxicol Sci 103:125-136.

Condé F, Lund JS, Jacobowitz DM, Baimbridge KG, Lewis DA (1994) Local circuit neurons immunoreactive for calretinin, calbindin D-28k or parvalbumin in monkey prefrontal cortex: distribution and morphology. J Comp Neurol 341:95-116.

Conti L, Pollard SM, Gorba T, Reitano E, Toselli M, Biella G, Sun Y, Sanzone S, Ying QL, Cattaneo E, Smith A (2005) Nicheindependent symmetrical self-renewal of a mammalian tissue stem cell. PLoS Biol 3:e283.

Dachet F, Bagla S, Keren-Aviram G, Morton A, Balan K, Saadat L, Valyi-Nagy T, Kupsky W, Song F, Dratz E, Loeb JA (2015) Predicting novel histopathological microlesions in human epileptic brain through transcriptional clustering. Brain 138:356-370. 
del Río MR, DeFelipe J (1996) Colocalization of calbindin D-28k, calretinin, and GABA immunoreactivities in neurons of the human temporal cortex. J Comp Neurol 369:472-482.

Devlin AC, Burr K, Borooah S, Foster JD, Cleary EM, Geti I, Vallier L, Shaw CE, Chandran S, Miles GB (2015) Human iPSC-derived motoneurons harbouring TARDBP or C9ORF72 ALS mutations are dysfunctional despite maintaining viability. Nat Commun 6:5999.

Fernandez-Salguero PM, Hilbert DM, Rudikoff S, Ward JM, Gonzalez FJ (1996) Aryl-hydrocarbon receptor-deficient mice are resistant to 2,3,7,8-tetrachlorodibenzo-p-dioxin-induced toxicity. Toxicol Appl Pharmacol 140:173-179.

Francis F, Meyer G, Fallet-Bianco C, Moreno S, Kappeler C, Socorro AC, Tuy FP, Beldjord C, Chelly J (2006) Human disorders of cortical development: from past to present. Eur J Neurosci 23: 877-893.

Gabbott PL, Bacon SJ (1996) Local circuit neurons in the medial prefrontal cortex (areas 24a,b,c, 25 and 32) in the monkey: II. Quantitative areal and laminar distributions. J Comp Neurol 364: 609-636.

Glaser T, Pollard SM, Smith A, Brüstle O (2007) Tripotential differentiation of adherently expandable neural stem (NS) cells. PLoS One 2:e298.

Gohlke JM, Stockton PS, Sieber S, Foley J, Portier CJ (2009) AhRmediated gene expression in the developing mouse telencephaIon. Reprod Toxicol 28:321-328.

Gomez A, Bindesbøll C, Satheesh SV, Grimaldi G, Hutin D, MacPherson L, Ahmed S, Tamblyn L, Cho T, Nebb HI, Moen A, Anonsen $\mathrm{JH}$, Grant DM, Matthews J (2018) Characterization of TCDDinducible poly-ADP-ribose polymerase (TIPARP/ARTD14) catalytic activity. Biochem J 475:3827-3846.

Grimaldi G, Rajendra S, Matthews J (2018) The aryl hydrocarbon receptor regulates the expression of TIPARP and its cis long non-coding RNA, TIPARP-AS1. Biochem Biophys Res Commun 495:2356-2362.

Han B, Zhang Y, Zhang Y, Bai Y, Chen X, Huang R, Wu F, Leng S, Chao J, Zhang JH, Hu G, Yao H (2018) Novel insight into circular RNA HECTD1 in astrocyte activation via autophagy by targeting MIR142-TIPARP: implications for cerebral ischemic stroke. Autophagy 14:1164-1184

Hans F, Dimitrov S (2001) Histone H3 phosphorylation and cell division. Oncogene 20:3021-3027.

Härtig W, Brauer K, Brückner G (1992) Wisteria floribunda agglutininlabelled nets surround parvalbumin-containing neurons. Neuroreport 3:869-872.

Hood DB, Woods L, Brown L, Johnson S, Ebner FF (2006) Gestational 2,3,7,8-tetrachlorodibenzo-p-dioxin exposure effects on sensory cortex function. Neurotoxicology 27:1032-1042.

Huang X, Powell-Coffman JA, Jin Y (2004) The AHR-1 aryl hydrocarbon receptor and its co-factor the AHA-1 aryl hydrocarbon receptor nuclear translocator specify GABAergic neuron cell fate in C. elegans. Development 131:819-828.

Huang Y, Song NN, Lan W, Hu L, Su CJ, Ding YQ, Zhang L (2013) Expression of transcription factor Satb2 in adult mouse brain. Anat Rec (Hoboken) 296:452-461.

Hutin D, Grimaldi G, Matthews J (2018a) Methods to study TCDDinducible poly-ADP-ribose polymerase (TIPARP) mono-ADPribosyltransferase activity. Methods Mol Biol 1813:109-124.

Hutin D, Tamblyn L, Gomez A, Grimaldi G, Soedling H, Cho T, Ahmed S, Lucas C, Kanduri C, Grant DM, Matthews J (2018b) Hepatocyte-specific deletion of TIPARP, a negative regulator of the aryl hydrocarbon receptor, is sufficient to increase sensitivity to dioxin-induced wasting syndrome. Toxicol Sci 165:347-360.

Keays DA, Tian G, Poirier K, Huang GJ, Siebold C, Cleak J, Oliver PL, Fray M, Harvey RJ, Molnár Z, Piñon MC, Dear N, Valdar W, Brown SD, Davies KE, Rawlins JN, Cowan NJ, Nolan P, Chelly J, Flint J (2007) Mutations in alpha-tubulin cause abnormal neuronal migration in mice and lissencephaly in humans. Cell 128:45-57.

Kerjan G, Gleeson JG (2007) Genetic mechanisms underlying abnormal neuronal migration in classical lissencephaly. Trends Genet 23:623-630.
Kimura E, Tohyama C (2017) Embryonic and postnatal expression of aryl hydrocarbon receptor mRNA in mouse brain. Front Neuroanat $11: 4$.

Kimura E, Kubo K, Matsuyoshi C, Benner S, Hosokawa M, Endo T, Ling W, Kohda M, Yokoyama K, Nakajima K, Kakeyama M, Tohyama C (2015) Developmental origin of abnormal dendritic growth in the mouse brain induced by in utero disruption of aryl hydrocarbon receptor signaling. Neurotoxicol Teratol 52:42-50.

Kimura E, Kubo KI, Endo T, Ling W, Nakajima K, Kakeyama M, Tohyama C (2017) Impaired dendritic growth and positioning of cortical pyramidal neurons by activation of aryl hydrocarbon receptor signaling in the developing mouse. PLoS One 12:e0183497.

Kolluri SK, Weiss C, Koff A, Göttlicher M (1999) p27(Kip1) induction and inhibition of proliferation by the intracellular Ah receptor in developing thymus and hepatoma cells. Genes Dev 13:17421753.

Kosaka T, Heizmann CW (1989) Selective staining of a population of parvalbumin-containing GABAergic neurons in the rat cerebral cortex by lectins with specific affinity for terminal $\mathrm{N}$-acetylgalactosamine. Brain Res 483:158-163.

Kozaki T, Komano J, Kanbayashi D, Takahama M, Misawa T, Satoh T, Takeuchi O, Kawai T, Shimizu S, Matsuura Y, Akira S, Saitoh T (2017) Mitochondrial damage elicits a TCDD-inducible poly(ADPribose) polymerase-mediated antiviral response. Proc Natl Acad Sci USA 114:2681-2686.

Kumar RA, Pilz DT, Babatz TD, Cushion TD, Harvey K, Topf M, Yates L, Robb S, Uyanik G, Mancini GM, Rees MI, Harvey RJ, Dobyns WB (2010) TUBA1A mutations cause wide spectrum lissencephaly (smooth brain) and suggest that multiple neuronal migration pathways converge on alpha tubulins. Hum Mol Genet 19:2817-2827.

Larsen SC, Hendriks IA, Lyon D, Jensen LJ, Nielsen ML (2018) Systems-wide analysis of serine ADP-ribosylation reveals widespread occurrence and site-specific overlap with phosphorylation. Cell Rep 24:2493-2505.e4.

Li L, Wei D, Wang Q, Pan J, Liu R, Zhang X, Bao L (2012) MEC-17 deficiency leads to reduced $\alpha$-tubulin acetylation and impaired migration of cortical neurons. J Neurosci 32:12673-12683.

Li MD, Burns TC, Morgan AA, Khatri P (2014) Integrated multi-cohort transcriptional meta-analysis of neurodegenerative diseases. Acta Neuropathol Commun 2:93.

Lian G, Sheen VL (2015) Cytoskeletal proteins in cortical development and disease: actin associated proteins in periventricular heterotopia. Front Cell Neurosci 9:99.

Liang CC, Park AY, Guan JL (2007) In vitro scratch assay: a convenient and inexpensive method for analysis of cell migration in vitro. Nat Protoc 2:329-333.

Lin $\mathrm{CH}$, Juan SH, Wang CY, Sun YY, Chou CM, Chang SF, Hu SY, Lee WS, Lee YH (2008) Neuronal activity enhances aryl hydrocarbon receptor-mediated gene expression and dioxin neurotoxicity in cortical neurons. J Neurochem 104:1415-1429.

Ma Q, Baldwin KT, Renzelli AJ, McDaniel A, Dong L (2001) TCDDinducible poly(ADP-ribose) polymerase: a novel response to 2,3,7,8-tetrachlorodibenzo-p-dioxin. Biochem Biophys Res Commun 289:499-506.

MacPherson L, Tamblyn L, Rajendra S, Bralha F, McPherson JP, Matthews J (2013) 2,3,7,8-Tetrachlorodibenzo-p-dioxin poly(ADPribose) polymerase (TiPARP, ARTD14) is a mono-ADPribosyltransferase and repressor of aryl hydrocarbon receptor transactivation. Nucleic Acids Res 41:1604-1621.

Magiera MM, Janke C (2014) Post-translational modifications of tubulin. Curr Biol 24:R351-R354.

Manzini MC, Walsh CA (2011) What disorders of cortical development tell us about the cortex: one plus one does not always make two. Curr Opin Genet Dev 21:333-339.

Matsuo R, Murayama A, Saitoh Y, Sakaki Y, Inokuchi K (2000) Identification and cataloging of genes induced by long-lasting long-term potentiation in awake rats. J Neurochem 74:2239-2249.

Mitsuhashi T, Yonemoto J, Sone H, Kosuge Y, Kosaki K, Takahashi $\mathrm{T}$ (2010) In utero exposure to dioxin causes neocortical dysgenesis 
through the actions of p27Kip1. Proc Natl Acad Sci USA 107: 16331-16335.

Nguyen MN, Nishijo M, Nguyen AT, Bor A, Nakamura T, Hori E, Nakagawa H, Ono T, Nishijo H (2013) Effects of maternal exposure to 2,3,7,8-tetrachlorodibenzo-p-dioxin on parvalbumin- and calbindin-immunoreactive neurons in the limbic system and superior colliculus in rat offspring. Toxicology 314:125-134.

Nishijo M, Kuriwaki J, Hori E, Tawara K, Nakagawa H, Nishijo H (2007) Effects of maternal exposure to 2,3,7,8-tetrachlorodibenzop-dioxin on fetal brain growth and motor and behavioral development in offspring rats. Toxicol Lett 173:41-47.

Pelletier B, Dhainaut F, Pauly A, Zahnd JP (1988) Evaluation of growth rate in adhering cell cultures using a simple colorimetric method. J Biochem Biophys Methods 16:63-73.

Perera FP, Li Z, Whyatt R, Hoepner L, Wang S, Camann D, Rauh V (2009) Prenatal airborne polycyclic aromatic hydrocarbon exposure and child IQ at age 5 years. Pediatrics 124:e195-e202.

Pollard SM (2013) In vitro expansion of fetal neural progenitors as adherent cell lines. Methods Mol Biol 1059:13-24.

Powers BE, Lin TM, Vanka A, Peterson RE, Juraska JM, Schantz SL (2005) Tetrachlorodibenzo-p-dioxin exposure alters radial arm maze performance and hippocampal morphology in female AhR mice. Genes Brain Behav 4:51-59.

Qin H, Powell-Coffman JA (2004) The Caenorhabditis elegans aryl hydrocarbon receptor, AHR-1, regulates neuronal development. Dev Biol 270:64-75.

Roper SJ, Chrysanthou S, Senner CE, Sienerth A, Gnan S, Murray A, Masutani M, Latos P, Hemberger M (2014) ADP- ribosyltransferases Parp1 and Parp7 safeguard pluripotency of ES cells. Nucleic Acids Res 42:8914-8927.

Schindelin J, Arganda-Carreras I, Frise E, Kaynig V, Longair M, Pietzsch T, Preibisch S, Rueden C, Saalfeld S, Schmid B, Tinevez JY, White DJ, Hartenstein V, Eliceiri K, Tomancak P, Cardona A (2012) Fiji: an open-source platform for biological-image analysis. Nat Methods 9:676-682.

Schmahl J, Rizzolo K, Soriano P (2008) The PDGF signaling pathway controls multiple steroid-producing lineages. Genes Dev 22:32553267.

Seegal RF (1996) Epidemiological and laboratory evidence of PCBinduced neurotoxicity. Crit Rev Toxicol 26:709-737.

Steenland HW (2010) Mouse medial-prefrontal cortex involvement in trace fear memory during wakefulness and sleep. PhD thesis, University of Toronto.

Stockinger B, Meglio PD, Gialitakis M, Duarte JH (2014) The aryl hydrocarbon receptor: multitasking in the immune system. Annu Rev Immunol 32:403-432.

Tsiarli MA (2014) Dissecting the role of the glucocorticoid receptor in embryonic cortical development: insights from in utero glucocorticoid exposure. PhD thesis, University of Pittsburgh.

Vyas S, Chesarone-Cataldo M, Todorova T, Huang YH, Chang P (2013) A systematic analysis of the PARP protein family identifies new functions critical for cell physiology. Nat Commun 4:2240.

Yamada T, Horimoto H, Kameyama T, Hayakawa S, Yamato H, Dazai M, Takada A, Kida H, Bott D, Zhou AC, Hutin D, Watts TH, Asaka M, Matthews J, Takaoka A (2016) Constitutive aryl hydrocarbon receptor signaling constrains type I interferon-mediated antiviral innate defense. Nat Immunol 17:687-694. 